\title{
From Dynamic Modeling to Experimentation of Induction Motor Powered by Doubly-Fed Induction Generator by Passivity-Based Control
}

\author{
M. Becherif ${ }^{1}$, A. Bensadeq ${ }^{2}$, E. Mendes ${ }^{3}$, A. Henni ${ }^{4}$, \\ P. Lefley ${ }^{5}$ and M.Y Ayad ${ }^{6}$ \\ ${ }^{1}$ UTBM, FEMTO-ST/FCLab, UMR CNRS 6174, 90010 Belfort Cedex \\ ${ }^{2}$ AElectrical Power \& Power Electronics Group, Department of Engineering \\ ${ }^{3}$ Grenoble INP - LCIS/ESISAR, BP 5426902 Valence Cedex 9 \\ ${ }^{4}$ Alstom Power - Energy Business Management \\ ${ }^{5}$ Electrical Power \& Power Electronics Group, Department of Engineering \\ University of Leicester \\ ${ }^{6}$ IEEE Member \\ $1,3,4,6$ France \\ ${ }^{2,5} \mathrm{UK}$
}

\section{Introduction}

DFIG wind turbines are nowadays more widely used especially in large wind farms. The main reason for their popularity when connected to the electrical network is their ability to supply power at constant voltage and frequency while the rotor speed varies, which makes it suitable for applications with variable speed, see for instance (10), (11). Additionally, when a bidirectional AC-AC converter is used in the rotor circuit, the speed range can be extended above its synchronous value recovering power in the regenerative operating mode of the machine. The DFIG concept also provides the possibility to control the overall system power factor. A DFIG wind turbine utilizes a wound rotor that is supplied from a frequency converter, providing speed control together with terminal voltage and power factor control for the overall system.

DFIGs have been traditionally used to convert mechanical power into electrical power operating near synchronous speed. Some advantages of DFIGs over synchronous or squirrel cage generators include the high overall efficiency of the system and the low power rating of the converter, which is only rated by the maximum rotor voltage and current. In a typical scenario the prime mover is running at constant speed, and the main concern is the static optimization of the power flow from the primary energy source to the grid. A good introduction to the operational characteristic of the grid connected DFIG can be found in (5). We consider in this paper the isolated operation of a DFIG driven by a prime mover, with its stator connected to a load-which is in this case an IM. Isolated generating units are economically attractive, hence increasingly popular, in the new era of the deregulated market. The possibility of a DFIG supplying an isolated load has been indicated in (6), (7) where some 
mention is made of the steady-state control problem. In (8) a system is presented in which the rotor is supplied from a battery via a PWM converter with experimental results from a $200 \mathrm{~W}$ prototype. A control system based on regulating the rms voltage of the DFIG is used which results in large voltage deviations and very slow recovery following load changes. See also $(9 ; 12)$ where feedback linearization and sliding mode principles are used for the design of the motor speed controller.

This paper presents a dynamic model of the DFIG-IM and proves that this system is Blondel-Park transformable. It is also shown that the zero dynamics is unstable for a certain operating regime. We implemented the passivity-based controller (PBC) that we proposed in (3) to a $200 \mathrm{~W}$ DFIG interconnected with an IM prototype available in IRII-UPC (Institute of Robotics and Industrial Informatics - University Polytechnic of Catalonia). The setup is controlled using a computer running RT-Linux. The whole system is decomposed in a mechanical subsystem which plays the role of the mechanical speed loop, controlled by a classical PI and an electrical subsystem controlled by the PBC where the model inversion was used to build a reference model.

The proposed PBC achieves the tracking control of the IM mechanical rotor speed and flux norm, the practical advantage of the PBC consists of using only the measurements of the two mechanical coordinates (Motor and Generator positions). The experiments have shown that the $\mathrm{PBC}$ is robust to variations in the machines' parameters.

In addition to the PBC applied to the electrical subsystem, we proposed a classical PI controller, where the rotor voltage control law is obtained via a control of the stator currents toward their desired values, those latter are obtained by the inversion of the model.

In the sequel, and for the control of the electrical subsystem a combination of the PBC + Proportional action for the control of the stator currents is applied. The last controller is a combination of PBC + PI action for the control of the stator currents.

The stability analysis is presented. The simulations and practical results show the effectiveness of the proposed solutions, and robustness tests on account of variations in the machines' parameters are also presented to highlight the performance of the different controllers.

The main disadvantage of the DFIG is the slip rings, which reduce the life time of the machine and increases the maintenance costs. To overcome this drawback an alternative machine arrangement is proposed, in section 6, which is the Brushless Doubly Fed twin Induction Generator (BDFTIG). The system is anticipated as an advanced solution to the conventional doubly fed induction generator (DFIG) to decrease the maintenance cost and develop the system reliability of the wind turbine system. The proposed BDFTIG employs two cascaded induction machines each consisting of two wound rotors, connected in cascade to eliminate the brushes and copper rings in the DFIG. The dynamic model of BDFTIG with two machines' rotors electromechanically coupled in the back-to-back configuration is developed and implemented using Matlab/Simulink.

\section{System configuration and mathematical model}

The configuration of the system considered in this paper is depicted in Fig.1. It consists of a wound rotor DFIG, a squirrel cage IM and an external mechanical device that can supply or extract mechanical power, e.g., a flywheel inertia. The stator windings of the IM are connected to the stator windings of the generator whose rotor voltage is regulated by a bidirectional converter. The electrical equivalent circuit is shown in Fig. 2. The main interest in this 
configuration is that it permits a bidirectional power flow between the motor, which may operate in regenerative mode, and the generator.

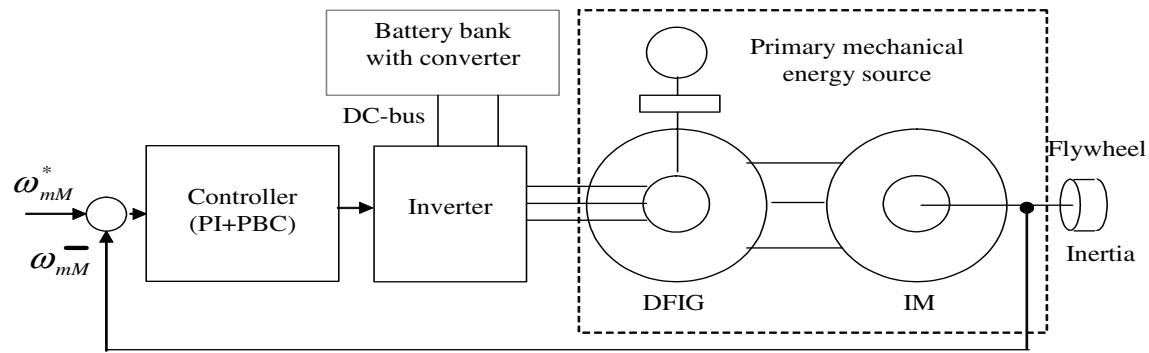

Fig. 1. System configuration with speed controller.

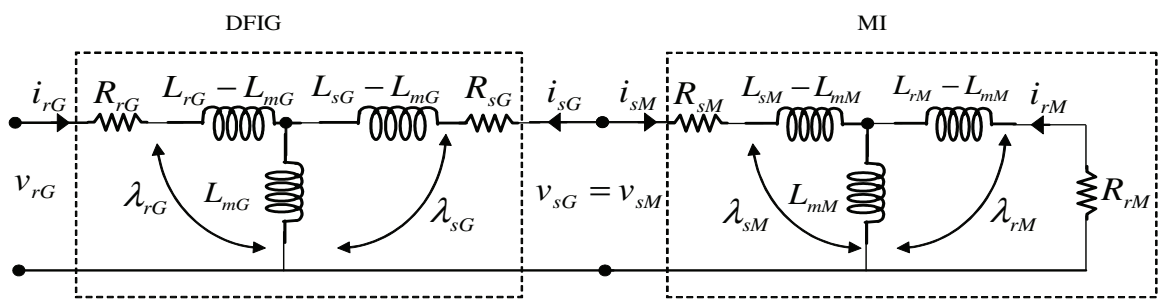

Fig. 2. Equivalent circuit of the DFIG with IM.

In Fig. 3, we show a power port viewpoint description of the system. The DFIG is a three-port system with conjugated power port variables ${ }^{1}$ prime mover torque and speed, $\left(\tau_{L G}, \omega_{G}\right)$, and rotor and stator voltages and currents, $\left(v_{r G}, i_{r G}\right),\left(v_{s G}, i_{s G}\right)$, respectively. The IM, on the other hand, is a two-port system with port variables motor load torque and speed, $\left(\tau_{L M}, \omega_{M}\right)$, and stator voltages and currents. The DFIG and the IM are coupled through the interconnection

$$
\begin{aligned}
v_{S G} & =v_{S M} \\
i_{S G} & =-i_{S M} .
\end{aligned}
$$

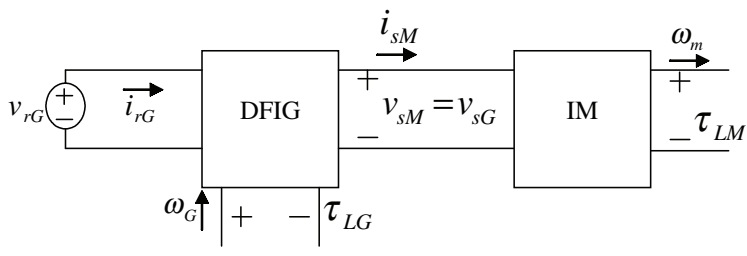

Fig. 3. Power port representation of the DFIG with IM.

To obtain the mathematical model of the overall system ideal symmetrical phases with uniform air-gap and sinusoidally distributed phase windings are assumed. The permeability

\footnotetext{
${ }^{1}$ The qualifier "conjugated power" is used to stress the fact that the product of the port variables has the units of power.
} 
of the fully laminated cores is assumed to be infinite, and saturation, iron losses, end winding and slot effects are neglected. Only linear magnetic materials are considered, and it is further assumed that all parameters are constant and known. Under these assumptions, the voltage balance equations for the machines are

$$
\begin{aligned}
\dot{\lambda}_{s G}+R_{s G} i_{s G} & =v_{s G} \\
\dot{\lambda}_{r G}+R_{r G} i_{r G} & =v_{r G} \\
\dot{\lambda}_{s M}+R_{s M} i_{s M} & =v_{s M} \\
\dot{\lambda}_{r M}+R_{r M} i_{r M} & =0
\end{aligned}
$$

where $\lambda_{s G}, \lambda_{r G}\left(\lambda_{s M}, \lambda_{r M}\right)$ are the stator and rotor fluxes of the DFIG (IM, resp.), $L_{s G}, L_{r G}, L_{m G}$ $\left(L_{s M}, L_{r M}, L_{m M}\right)$ are the stator, rotor, and mutual inductances of the DFIG (IM, resp.); $R_{s G}, R_{r G}$ $\left(R_{s M}, R_{r M}\right)$ are the stator and rotor resistances of the DFIG (IM, resp.).

The interconnection (1) induces an order reduction in the system. To eliminate the redundant coordinates, and preserving the structure needed for application of the $\mathrm{PBC}$, we define

$$
\lambda_{s G M}=\lambda_{s G}-\lambda_{s M}
$$

which upon replacement in the equations above, and with some simple manipulations, yields the equation

$$
\dot{\lambda}+R i=B v_{r G}
$$

where we have defined the vector signals

$$
\lambda=\left[\begin{array}{c}
\lambda_{r G} \\
\lambda_{s G M} \\
\lambda_{r M}
\end{array}\right], \quad i=\left[\begin{array}{c}
i_{r G} \\
i_{s G} \\
i_{r M}
\end{array}\right]
$$

and the resistance and input matrices

$$
R=\operatorname{diag}\{\underbrace{R_{r G}}_{R_{1}} I_{2}, \underbrace{\left(R_{s G}+R_{s M}\right)}_{R_{2}} I_{2}, \underbrace{R_{r M}}_{R_{3}} I_{2}\}, \quad B=\left[\begin{array}{ll}
I_{2} & 0
\end{array}\right]^{T} \in \mathbb{R}^{6 \times 2}
$$

To complete the model of the electrical subsystem, we recall that fluxes and currents are related through the inductance matrix by

$$
\lambda=L(\theta) i,
$$

where the latter takes in this case the form

$$
L(\theta)=\left[\begin{array}{ccc}
L_{r G} I_{2} & L_{m G} e^{-J n_{G} \theta_{G}} & 0 \\
L_{m G} e^{J n_{G} \theta_{G}} & \left(L_{s G}+L_{s M}\right) I_{2} & -L_{m M} e^{J n_{M} \theta_{M}} \\
0 & -L_{m M} e^{-J n_{M} \theta_{M}} & L_{r M} I_{2}
\end{array}\right]
$$

where $n_{G}, n_{M}$ denote the number of pole pairs, $\theta_{G}, \theta_{M}$ the mechanical rotor positions (with respect to the stator) and to simplify the notation we have introduced

$$
\theta=\left[\begin{array}{l}
\theta_{G} \\
\theta_{M}
\end{array}\right], \quad J=\left[\begin{array}{cc}
0 & -1 \\
1 & 0
\end{array}\right]=-J^{T}, \quad e^{J x}=\left[\begin{array}{cc}
\cos (x) & -\sin (x) \\
\sin (x) & \cos (x)
\end{array}\right]=\left(e^{-J x}\right)^{T}
$$




$$
\begin{aligned}
L^{-1}(\theta) & =\frac{1}{\Delta}\left[\begin{array}{ccc}
{\left[L_{r M}\left(L_{S G}+L_{s M}\right)-L_{m M}^{2}\right] I_{2}} & -L_{m G} L_{r M} e^{-J n_{G} \theta_{G}} & -L_{m G} L_{m M} e^{-J\left(n_{G} \theta_{G}-n_{M} \theta_{M}\right)} \\
-L_{m G} L_{r M} e^{J n_{G} \theta_{G}} & L_{r G} L_{r M} I_{2} & L_{r G} L_{m M} e^{J n_{M} \theta_{M}} \\
-L_{m G} L_{m M} e^{J\left(n_{G} \theta_{G}-n_{M} \theta_{M}\right)} & L_{r G} L_{m M} e^{-J n_{M} \theta_{M}} & {\left[L_{r G}\left(L_{s G}+L_{s M}\right)-L_{m G}^{2}\right] I_{2}}
\end{array}\right] \\
& \triangleq \frac{1}{\Delta}\left[\begin{array}{ccc}
L_{11}^{\prime} & L_{12}^{\prime} & L_{13}^{\prime} \\
L_{12}^{\prime T} & L_{22}^{\prime} & L_{23}^{\prime} \\
L_{11}^{\prime T} & L_{23}^{\prime T} & L_{33}^{\prime}
\end{array}\right]
\end{aligned}
$$

where

$$
\Delta=L_{r G}\left[L_{r M}\left(L_{s G}+L_{s M}\right)-L_{m M}^{2}\right]-L_{r M} L_{m G}^{2}<0
$$

We recall that, due to physical considerations, $R>0, L(\theta)=L^{T}(\theta)>0$ and $L^{-1}(\theta)=$ $L^{-1^{T}}(\theta)>0$.

A state-space model of the (6-th order) electrical subsystem is finally obtained replacing (7) in (6) as

$$
\Sigma_{e}: \dot{\lambda}+R L(\theta)^{-1} \lambda=B v_{r G}
$$

The mechanical dynamics are obtained from Newton's second law and are given by

$$
\Sigma_{m}: J_{m} \ddot{\theta}+B_{m} \dot{\theta}=\tau-\tau_{L}
$$

where $J_{m}=\operatorname{diag}\left\{J_{G}, J_{M}\right\}>0$ is the mechanical inertia matrix, $B_{m}=\operatorname{diag}\left\{B_{G}, B_{M}\right\} \geq 0$ contains the damping coefficients, $\tau_{L}=\left[\tau_{L G}, \tau_{L M}\right]^{T}$ are the external torques, that we will assume constant in the sequel. The generated torques are calculated as usual from

$$
\tau=\left[\begin{array}{c}
\tau_{G} \\
\tau_{M}
\end{array}\right]=-\frac{1}{2} \frac{\partial}{\partial \theta}\left(\lambda^{T}[L(\theta)]^{-1} \lambda\right) .
$$

From (7), we obtain the alternative expression

$$
\tau=\frac{1}{2} \frac{\partial}{\partial \theta}\left(i^{T} L(\theta) i\right)
$$

The following equivalent representations of the torques, that are obtained from direct calculations using (7), (8) and (14), will be used in the sequel

$$
\begin{aligned}
\tau & =\left[\begin{array}{c}
-L_{m G} i_{r G}^{T} J e^{-J n_{G} \theta_{G}} i_{s G} \\
-L_{m M} i_{s G}^{T} J e^{J n_{M} \theta_{M}} i_{r M}
\end{array}\right] \\
& =\left[\begin{array}{c}
-\frac{n_{G}}{R_{s G}+R_{s M}} \dot{\lambda}_{s G M}^{T} J\left(\lambda_{s G M}-L_{m M} e^{J n_{M} \theta_{M}} i_{r M}\right) \\
\frac{n_{M}}{R_{r M}} \dot{\lambda}_{r M}^{T} J \lambda_{r M}
\end{array}\right]
\end{aligned}
$$

\subsection{Modeling of the DFIG-IM in the stator frame of the two machines}

It has been shown in (4) and (3) that the DFIG-IM is Blondel-Park transformable using the following rotating matrix:

$$
\operatorname{Rot}\left(\sigma, \theta_{G}, \theta_{M}\right)=\left[\begin{array}{ccc}
e^{(J \sigma)} & 0 & 0 \\
0 & e^{\left(J\left(\sigma+n_{G} \theta_{G}\right)\right)} & 0 \\
0 & 0 & e^{\left(J\left(\sigma+n_{G} \theta_{G}-n_{M} \theta_{M}\right)\right)}
\end{array}\right]
$$


where $\sigma$ is an arbitrary angle.

The model of the DFIG-IM in the stator frame of the two machines is given by (see (4) and (3) for in depth details):

$\left\{\begin{array}{c}{\left[\begin{array}{c}\dot{\hat{\lambda}}_{r G} \\ \dot{\lambda}_{s M G} \\ \hat{\hat{\lambda}}_{r M}\end{array}\right]+\left[\begin{array}{ccc}a I_{2}-n_{G} \dot{\theta}_{G} J & b I_{2} & 0 \\ a I_{2}-n_{G} \dot{\theta}_{G} J & e I_{2} & -c I_{2}+n_{M} \dot{\theta}_{M} J \\ 0 & -d I_{2} & c I_{2}-n_{M} \dot{\theta}_{M} J\end{array}\right]\left[\begin{array}{c}\widehat{\lambda}_{r G} \\ \lambda_{s M G} \\ \hat{\lambda}_{r M}\end{array}\right]=\left[\begin{array}{c}I_{2} \\ I_{2} \\ 0\end{array}\right] \widehat{v}_{r G}} \\ {\left[\begin{array}{c}J_{G} \dot{\omega}_{G} \\ J_{M} \dot{\omega}_{M}\end{array}\right]+\left[\begin{array}{cc}B_{G} & 0 \\ 0 & B_{M}\end{array}\right]\left[\begin{array}{c}\omega_{G} \\ \omega_{M}\end{array}\right]+\left[\begin{array}{c}f \lambda_{s M G}^{T} J \widehat{\lambda}_{r G} \\ -f \lambda_{s M G}^{T} J \hat{\lambda}_{r M}\end{array}\right]=\left[\begin{array}{c}-\tau_{L G} \\ -\tau_{L M}\end{array}\right]}\end{array}\right.$

or

$$
\left\{\begin{array}{c}
{\left[\begin{array}{c}
\dot{\hat{\lambda}}_{r G} \\
\dot{\lambda}_{s M G} \\
\dot{\hat{\lambda}}_{r M}
\end{array}\right]+\left(R+L_{G} n_{G} \dot{\theta}_{G}+L_{M} n_{M} \dot{\theta}_{M}\right)\left[\begin{array}{c}
\hat{i}_{r G} \\
i_{s M} \\
\hat{i}_{r M}
\end{array}\right]=\left[\begin{array}{c}
I_{2} \\
I_{2} \\
0
\end{array}\right] \widehat{v}_{r G}} \\
{\left[\begin{array}{c}
J_{G} \dot{\omega}_{G} \\
J_{M} \dot{\omega}_{M}
\end{array}\right]+\left[\begin{array}{cc}
B_{G} & 0 \\
0 & B_{M}
\end{array}\right]\left[\begin{array}{c}
\omega_{G} \\
\omega_{M}
\end{array}\right]+\left[\begin{array}{c}
f \lambda_{s M G}^{T} J \widehat{\lambda}_{r G} \\
-f \lambda_{s M G}^{T} J \widehat{\lambda}_{r M}
\end{array}\right]=\left[\begin{array}{c}
-\tau_{L G} \\
-\tau_{L M}
\end{array}\right]}
\end{array}\right.
$$

$\lambda_{S M G}$ corresponds to the total leakage flux of the two machines referred to the stators of the machines.

$L_{S M G}$ represent the total leakage inductance.

with

$$
\left\{\begin{array}{c}
R=\left[\begin{array}{ccc}
\widehat{R}_{r G} I_{2} & 0 & 0 \\
\widehat{R}_{r G} I_{2} & \left(R_{s G}+R_{s M}\right) I_{2} & -\widehat{R}_{r M} I_{2} \\
0 & 0 & \widehat{R}_{r M} I_{2}
\end{array}\right] \\
L_{G}=L_{M G}\left[\begin{array}{ccc}
-J & J & 0 \\
-J & J & 0 \\
0 & 0 & 0
\end{array}\right] \text { et } L_{M}=L_{M M}\left[\begin{array}{ccc}
0 & 0 & 0 \\
0 & J & J \\
0 & -J & -J
\end{array}\right]
\end{array}\right.
$$

with the positive parameters: $a=\widehat{R}_{r G} L_{M G^{\prime}}^{-1}, b=\widehat{R}_{r G} L_{s M G^{\prime}}^{-1} c=\widehat{R}_{r M} L_{M M}^{-1}, d=\widehat{R}_{r M} L_{s M G^{\prime}}^{-1}$ $e=\left(\widehat{R}_{r G}+R_{s G}+R_{s M}+\widehat{R}_{r M}\right) L_{s M G^{\prime}}^{-1} f=L_{s M G^{\prime}}^{-1}$ and the following transformations:

$$
\begin{array}{ll}
\widehat{\lambda}_{r G}=\frac{L_{m G}}{L_{r G}} e^{J n_{G} \theta_{G}} \lambda_{r G}, & \widehat{v}_{r G}=\frac{L_{m G}}{L_{r G}} e^{J n_{G} \theta_{G}} v_{r G} \\
\widehat{\lambda}_{r M}=\frac{L_{m M}}{L_{r M}} e^{J n_{M} \theta_{M}} \lambda_{r M}, & \widehat{i}_{r G}=\frac{L_{r G}}{L_{m G}} e^{J n_{G} \theta_{G}} i_{r G}
\end{array}
$$

\section{Properties of the model}

In this section, we derive some passivity and geometric properties of the model that will be instrumental to carry out our controller design.

\subsection{Passivity}

An explicit power port representation of the DFIG interconnected to the IM is presented in Fig.4 


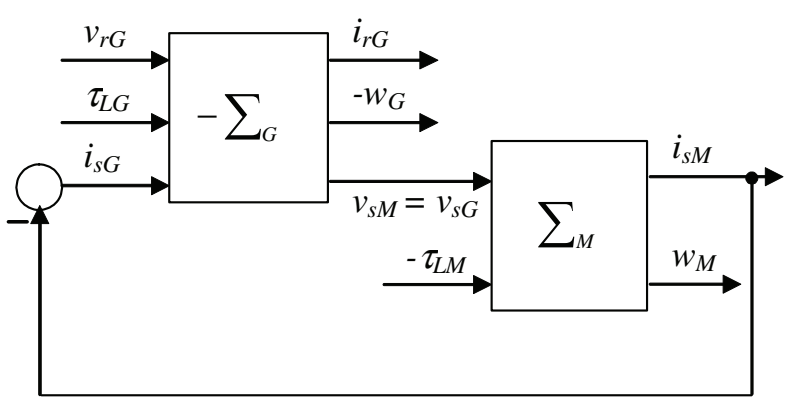

Fig. 4. Explicit power port representation of the DFIG with IM.

Claim 1. The interconnection of the DFIG with the IM presented in the explicit power port representation in Fig.4 is a passive system ${ }^{2}$ with the passive map $\left[\begin{array}{c}v_{r G} \\ \tau_{L G} \\ -\tau_{L M}\end{array}\right] \mapsto\left[\begin{array}{c}i_{r G} \\ -\omega_{G} \\ \omega_{M}\end{array}\right]$.

Proof. Consider the Fig. $4, \Sigma=\left[\begin{array}{c}\Sigma_{G} \\ \Sigma_{M}\end{array}\right]$ is passive $\Rightarrow\left[\begin{array}{c}v_{r G} \\ \tau_{L G} \\ -\tau_{L M}\end{array}\right] \mapsto\left[\begin{array}{c}i_{r G} \\ -\omega_{G} \\ \omega_{M}\end{array}\right]$ is passive?

For this purpose, we have to prove that $\int\left(v_{r G}^{T} i_{r G}-\tau_{L G} \omega_{G}-\tau_{L M} \omega_{M}\right) \geq 0$. We know that each machine separately is passive (see [1])

$$
\begin{aligned}
\Sigma_{G} \text { is a passive system } & \Leftrightarrow \int\left(v_{r G}^{T} i_{r G}-\tau_{L G} \omega_{G}+i_{S M}^{T} v_{S M}\right) \geq 0 \\
\Sigma_{M} \text { is a passive system } & \Leftrightarrow \int\left(v_{S G}^{T} i_{S G}-\tau_{L M} \omega_{M}\right) \geq 0
\end{aligned}
$$

where equation (1) has been used in (20) and (21). Let's consider

$$
d \triangleq \int\left(v_{r G}^{T} i_{r G}-\tau_{L G} \omega_{G}+i_{s M}^{T} v_{s M}\right) \geq 0
$$

Using the energy conserving principle $\int i_{S M}^{T} v_{s M}=-\int i_{S G}^{T} v_{S G}$ yields

$$
d=\int\left(v_{r G}^{T} i_{r G}-\tau_{L G} \omega_{G}\right)-\int i_{s G}^{T} v_{s G}
$$

From (21) we have

$$
-\int i_{S G}^{T} v_{S G} \leq-\int \tau_{L M} \omega_{M}
$$

Finally (23) and (24) yields

$$
\int\left(v_{r G}^{T} i_{r G}-\tau_{L G} \omega_{G}-\tau_{L M} \omega_{M}\right) \geq d \geq 0
$$

Hence, the passivity of the DFIG interconnected to the IM is proven

\footnotetext{
${ }^{2}$ Passive systems are defined here with no causality relation assumed among the port variables (13). This, more natural, definition is more suitable for applications where power flow (and not signal behaviour) is the primary concern.
} 


\section{Zero dynamics}

For the IM speed control, we are interested in the internal behaviour of the system when the motor torque $\tau_{M}$ is constant. In addition, for practical considerations, we are interested in the control of the IM flux norm $\left|\lambda_{r M}\right|$, where $|\cdot|$ is the Euclidean norm.

For the study of the zero dynamics regarding these two outputs, we consider the DFIG-IM model $^{3}$ given by (18).

The control $\widehat{v}_{r G}$ is determined to obtain the desired equilibrium points of the DFIG-IM:

$$
\begin{gathered}
\ddot{\theta}_{G}=\ddot{\theta}_{M}=0, \quad \dot{\theta}_{G}=\dot{\theta}_{G}^{d}=\text { Constant }, \quad \dot{\theta}_{M}=\dot{\theta}_{M}^{d}=\text { Constant }, \\
\tau_{L G}=\tau_{L G 0}=\text { Constant }, \quad \tau_{L M}=\tau_{L M 0}=\text { Constant } \\
\hat{\lambda}_{r M}^{T} \widehat{\lambda}_{r M}=\beta_{d}^{2}=\text { Constant }>0
\end{gathered}
$$

The IM mechanical dynamics show that the desired equilibrium points are obtained if:

$$
\tau_{M}=\tau_{M}^{d}=\tau_{L M 0}=\text { Constant }
$$

Hence:

$$
f \lambda_{s M G}^{T} J \widehat{\lambda}_{r M}=\tau_{M}=\tau_{M}^{d}=\text { Constant }
$$

The equation (27) can also be expressed by replacing $\lambda_{s M G}$ by its value given by the third line of the electrical subsystem (18):

$$
\frac{f}{d}\left(\dot{\widehat{\lambda}}_{r M}+c \widehat{\lambda}_{r M}-n_{M} \dot{\theta}_{M}^{d} J \widehat{\lambda}_{r M}\right)^{T} J \widehat{\lambda}_{r M}=\tau_{M}
$$

Hence

$$
\frac{f}{d}\left(\dot{\hat{\lambda}}_{r M}^{T} J \widehat{\lambda}_{r M}-n_{M} \dot{\theta}_{M}^{d} \widehat{\lambda}_{r M}^{T} \widehat{\lambda}_{r M}\right)=\frac{f}{d}\left(\dot{\hat{\lambda}}_{r M}^{T} J \widehat{\lambda}_{r M}-n_{M} \dot{\theta}_{M}^{d} \beta^{2}\right)=\tau_{M}=\tau_{M}^{d}=\text { cte }
$$

The relative degrees of the outputs $y_{1}=\beta^{2}=\widehat{\lambda}_{r M}^{T} \widehat{\lambda}_{r M}$ and $y_{2}=\tau_{M}=f \lambda_{s M G}^{T} J \widehat{\lambda}_{r M}$, regarding the input control $\widehat{v}_{r G}$, are 2 and 1 , respectively.

The zero dynamics of the mechanical subsystem (18) is stable, since the mechanical parameters are positive.

Following on we will analyze the zero dynamics of the electrical subsystem considering the equilibrium points such that (26), (27) and (28) are verified (we will omit the subscript $d$ ).

- Consequence of (26):

$$
\begin{aligned}
& \frac{d \beta^{2}}{d t}=0 \Rightarrow \dot{\hat{\lambda}}_{r M}^{T} \widehat{\lambda}_{r M}=0 \quad \text { et } \quad \widehat{\lambda}_{r M}^{T} \dot{\hat{\lambda}}_{r M}=0 \\
& 0=\hat{\lambda}_{r M}^{T} \dot{\hat{\lambda}}_{r M}=d \widehat{\lambda}_{r M}^{T} \lambda_{s M G}-c \widehat{\lambda}_{r M}^{T} \widehat{\lambda}_{r M}+n_{M} \dot{\theta}_{M} \widehat{\lambda}_{r M}^{T} J \widehat{\lambda}_{r M}
\end{aligned}
$$

with the electrical subsystem of (18), it comes: $0=d \widehat{\lambda}_{r M}^{T}\left(\lambda_{s M G}-\frac{c}{d} \widehat{\lambda}_{r M}\right)$

Hence, with (26), it comes as solution of $\lambda_{s M G}$ :

$$
\lambda_{s M G}=\frac{c}{d} \widehat{\lambda}_{r M}+\alpha J \widehat{\lambda}_{r M}, \quad \forall \alpha \in \mathbb{R}
$$

\footnotetext{
${ }^{3}$ The zero dynamics analysis is independent from the chosen frame.
} 
- Consequence of (27) and (28):

Replace (29) in (27):

$$
\tau_{M}=f \widehat{\lambda}_{r M}^{T}\left(\frac{c}{d} I_{2}-\alpha J\right) J \widehat{\lambda}_{r M}=f \alpha \widehat{\lambda}_{r M}^{T} \widehat{\lambda}_{r M}
$$

Since $\widehat{\lambda}_{r M}^{T} \widehat{\lambda}_{r M}=\beta^{2}=$ Constant:

$$
\tau_{M}=f \beta^{2} \alpha \Rightarrow \alpha=\frac{\tau_{M}}{f \beta^{2}}
$$

and with $\dot{\tau}_{M}=0$ :

$$
\dot{\tau}_{M}=f \beta^{2} \dot{\alpha} \Rightarrow \dot{\alpha}=0
$$

Recall of consequences of (26), (27) and (28):

At the equilibrium, the solutions of $\widehat{\lambda}_{r M}$ belong to the following set:

$$
\left\{\widehat{\lambda}_{r M} \in \mathbb{R}^{2} \mid \widehat{\lambda}_{r M}^{T} \widehat{\lambda}_{r M}=\beta^{2}>0, \widehat{\lambda}_{r M}^{T} \dot{\hat{\lambda}}_{r M}=0, \dot{\hat{\lambda}}_{r M}^{T} J \widehat{\lambda}_{r M}=\frac{d}{f} \tau_{M}+n_{M} \dot{\theta}_{M} \beta^{2}, \dot{\beta}=0\right\}
$$

At the equilibrium, the solutions of $\lambda_{s M G}$ belong to the following set:

$$
\left\{\lambda_{s M G} \in \mathbb{R}^{2} \mid \lambda_{s M G}=\frac{c}{d} \widehat{\lambda}_{r M}+\alpha J \widehat{\lambda}_{r M}, \alpha=\frac{\tau_{M}}{f \beta^{2}}, \dot{\alpha}=0\right\}
$$

Let take $\widehat{\lambda}_{r M}$ in the form :

$$
\widehat{\lambda}_{r M}=e^{J\left(\rho+n_{M} \theta_{M}\right)}\left[\begin{array}{l}
\beta \\
0
\end{array}\right]
$$

with the form (32) and the constrains of (30), it comes:

$$
\begin{gathered}
\dot{\beta}=0 \Rightarrow \dot{\hat{\lambda}}_{r M}=\left(\dot{\rho}+n_{M} \dot{\theta}_{M}\right) J \widehat{\lambda}_{r M} \text { and } \widehat{\lambda}_{r M}^{T} \dot{\hat{\lambda}}_{r M}=0 \\
\dot{\hat{\lambda}}_{r M}^{T} J \widehat{\lambda}_{r M}=\frac{d}{f} \tau_{M}+n_{M} \dot{\theta}_{M}^{d} \beta^{2} \Rightarrow\left(\dot{\rho}+n_{M} \dot{\theta}_{M}\right) \beta^{2}=\frac{d}{f} \tau_{M}+n_{M} \dot{\theta}_{M} \beta^{2} \Rightarrow \dot{\rho}=\frac{d}{f} \frac{\tau_{M}}{\beta^{2}}=d \alpha
\end{gathered}
$$

with $\alpha$ given by (31).

Hence the vectors $\lambda_{s M G}$ and $\hat{\lambda}_{r M}$ are completely defined by the outputs $y_{1}$ and $y_{2}$.

Analyzing the behaviour of the dynamics of the state $\widehat{\lambda}_{r G}$ :

the substraction of the two upper lines of (18) give:

$$
\dot{\hat{\lambda}}_{r G}=(b+e) \lambda_{s M G}+\dot{\lambda}_{s M G}-c \widehat{\lambda}_{r M}+n_{M} \dot{\theta}_{M} J \widehat{\lambda}_{r M}
$$

By replacing $\lambda_{s M G}$ by its value given by (31), the state becomes:

$$
\dot{\hat{\lambda}}_{r G}=\left[\frac{c(b+e-d)}{d} I_{2}+\left(\alpha(b+e)+n_{M} \dot{\theta}_{M}\right) J\right] \hat{\lambda}_{r M}+\left(\frac{c}{d} I_{2}+\alpha J\right) \dot{\hat{\lambda}}_{r M}
$$

Using the general form (32) and its derivative under the constrains (30), yields:

$$
\begin{aligned}
\dot{\hat{\lambda}}_{r G} & =\left[\left(\frac{c(b+e-d)}{d}-\alpha\left(\dot{\rho}+n_{M} \dot{\theta}_{M}\right)\right) I_{2}+\left(\alpha(b+e)+n_{M} \dot{\theta}_{M}+\left(\dot{\rho}+n_{M} \dot{\theta}_{M}\right) \frac{c}{d}\right) J\right] \widehat{\lambda}_{r M} \\
& =\left[c_{1} I_{2}+c_{2} J\right] \widehat{\lambda}_{r M}=M_{1} e r \widehat{\lambda}_{r M}
\end{aligned}
$$


with $M_{1}=\sqrt{c_{1}^{2}+c_{2}^{2}}=$ Constant and $\gamma=\arctan \frac{c 2}{c 1}=$ Constant if $c_{1} \neq 0$, else $\gamma=\frac{\pi}{2}$.

Then, with (32):

$$
\dot{\hat{\lambda}}_{r G}=M_{1} e^{J \gamma_{e}} e^{J\left(\rho+n_{M} \theta_{M}\right)}\left[\begin{array}{l}
\beta \\
0
\end{array}\right]=M_{1} \beta e^{J\left(\gamma+\rho+n_{M} \theta_{M}\right)}\left[\begin{array}{l}
1 \\
0
\end{array}\right]
$$

Consequently, $\widehat{\lambda}_{r G}$ is of the form:

$$
\widehat{\lambda}_{r G}=\frac{-M_{1} \beta}{\dot{\rho}+n_{M} \dot{\theta}_{M}} J e^{J\left(\gamma+\rho+n_{M} \theta_{M}\right)}\left[\begin{array}{l}
1 \\
0
\end{array}\right]+\text { Constant }
$$

because $\ddot{\rho}=\ddot{\theta}_{M}=0, \dot{\gamma}=0$ and $\dot{M}_{1}=0$.

We can then conclude that the dynamics of $\widehat{\lambda}_{r G}$ is stable if the desired operating point satisfies:

$$
\dot{\rho}+n_{M} \dot{\theta}_{M} \neq 0
$$

Consequently, the zero dynamics (the dynamics of $\widehat{\lambda}_{r G}$ ) is unstable when the desired operating point belongs to the slip line defined by:

$$
\dot{\rho}=-n_{M} \dot{\theta}_{M}
$$

or in terms of the controlled outputs $\beta^{2}$ and $\tau_{M}$ :

$$
\tau_{M}=-\frac{L_{r M}^{2}}{L_{m M}^{2} R_{r M}} n_{M} \dot{\theta}_{M} \beta^{2}
$$

With usual machine parameters, the operating point may belong to the slip line for very low speed which is not the case with the considered operating points

\section{Passivity-based controllers}

The PBC achieves the IM speed and rotor flux norm control with all internal signals remaining bounded under the condition $\dot{\rho}+n_{M} \dot{\theta}_{M} \neq 0$. From a practical point of view it is interesting to ensure the boundedness of the internal signals and in particular the stator current of the two machines. For this purpose, two classical controllers (Proportional and Proportional plus Integral) are applied or combined with the PBC on the stator current $i_{S G}$.

In this section we address the stability analysis of the following controllers:

1 PBC : Passivity Based control;

$2 \mathrm{PBC}+\mathrm{P}$ : Passivity Based control + Proportional action on the stator currents $i_{s G}$;

3 PBC + PI : Passivity Based control + Proportional plus Integral actions on the stator currents $i_{S G}$.

As defined in (3) a nested loop control configuration is adopted for the PBC control of the DFIG with the IM system. We propose to design first a torque tracking PBC for $\Sigma_{e}$, and then add a speed tracking loop around it. This leads to the nested-loop scheme depicted in Fig. 5 , where $C_{i l}$ is the inner-loop torque tracking $\mathrm{PBC}$ and $C_{o l}$ is an outer-loop speed controller, which generates the desired torque, and will be taken as a simple PI controller. The reader is referred to (1) for motivation and additional details on this control configuration. 


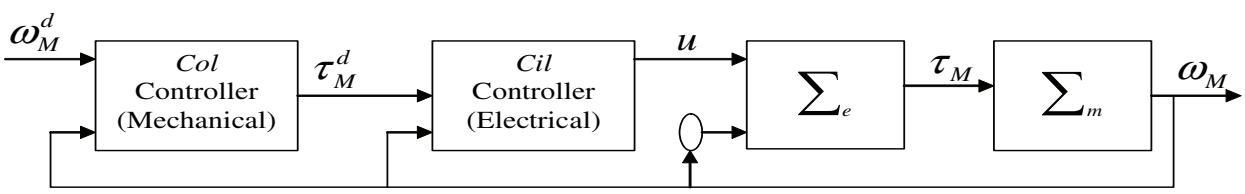

Fig. 5. Nested-loop control configuration.

\subsection{PBC}

To derive the torque tracking $\mathrm{PBC}$ we will shape the storage function $H_{\lambda}(\lambda)$, which has a minimum at zero, to take the form

$$
H_{\lambda}^{d}=\frac{1}{2} \tilde{\lambda}^{T} R^{-1} \tilde{\lambda} \geq 0
$$

where $\tilde{\lambda}=\lambda-\lambda^{d}$, with $\lambda^{d}$ a signal to be defined. As suggested in (1), we propose to establish the following relationship between $\lambda^{d}$ and $v_{r G}$ :

$$
B v_{r G}=\dot{\lambda}^{d}+R L^{-1}(\theta) \lambda^{d} .
$$

Comparing with (12) we see that this, so-called implicit representation of the controller, is a "copy" of the electrical subsystem but evaluated along some desired trajectories. We will prove now that this control action indeed shapes the storage function as desired. Combining (34) with (12) yields the error equation for the fluxes

$$
\dot{\tilde{\lambda}}+R L^{-1}(\theta) \tilde{\lambda}=0 .
$$

The derivative of the desired energy function (33) along the trajectory of (35) is

$$
\dot{H}_{\lambda}^{d}=-\tilde{\lambda}^{T} L^{-1} \tilde{\lambda} \leq 0
$$

Hence, $\tilde{\lambda}(t) \rightarrow 0$ exponentially.

To complete our torque tracking design there are two remaining issues:

(i) find an explicit representation for the controller (34);

(ii) select $\lambda^{d}$ such that, for any given desired trajectory $\tau_{M}^{*}(t)$, we have

$$
\tilde{\lambda}(t) \rightarrow 0 \Rightarrow \tau_{M}(t) \rightarrow \tau_{M}^{*}(t) ;
$$

\subsection{PBC + P}

The $\mathrm{PBC}+\mathrm{P}$ controller is given by the equation below:

$$
B v_{r G}=\dot{\lambda}^{d}+R L^{-1}(\theta) \lambda^{d}+B K_{p}\left(i_{s G}-i_{s G}^{d}\right)
$$

where $K_{p}$ is a proportional positive gain. We have:

$$
\begin{aligned}
i_{s G}-i_{s G}^{d} & =\frac{1}{\Delta}\left(L_{21}^{\prime}\left(\lambda_{r G}-\lambda_{r G}^{d}\right)+L_{22}^{\prime}\left(\lambda_{s G M}-\lambda_{s G M}^{d}\right)+L_{23}^{\prime}\left(\lambda_{r M}-\lambda_{r M}^{d}\right)\right) \\
& =\frac{1}{\Delta}\left[\begin{array}{lll}
L_{21}^{\prime} & L_{22}^{\prime} I_{2} & L_{23}^{\prime}
\end{array}\right](\underbrace{\lambda-\lambda^{d}}_{\tilde{\lambda}})=\underbrace{\left[\begin{array}{lll}
0 & I_{2} & 0
\end{array}\right]}_{P} L^{-1}(\theta) \tilde{\lambda}
\end{aligned}
$$

with $L_{i j}^{\prime}\left(i=1^{-} 3, j=1,3\right)$ and $\Delta$ are given by (10) and (11), respect. Then, 


$$
B v_{r G}=\dot{\lambda}^{d}+R L^{-1}(\theta) \lambda^{d}+K_{p} B P L^{-1}(\theta) \tilde{\lambda}
$$

The closed loop error dynamic can be written as the following:

$$
\dot{\tilde{\lambda}}=-R L^{-1}(\theta) \tilde{\lambda}-K_{p} B P L^{-1}(\theta) \tilde{\lambda}
$$

Consider the desired energy function given by (33), its derivative along the trajectories of (41) is:

$$
\begin{aligned}
\dot{H}_{\lambda}^{d} & =\tilde{\lambda}^{T} R^{-1} \dot{\tilde{\lambda}} \\
& =-\tilde{\lambda}^{T} \underbrace{L^{-1}(\theta)}_{>0} \tilde{\lambda}-\tilde{\lambda}^{T} \underbrace{R^{-1} B P L^{-1}(\theta)}_{\triangleq Q} \tilde{\lambda}
\end{aligned}
$$

To prove that the error dynamic (41) is stable, it's enough to prove that $Q$ is a positive semi-definite matrix:

$$
Q=R^{-1} B P L^{-1}(\theta)=\frac{1}{R_{1} \Delta}\left[\begin{array}{ccc}
L_{12}^{\prime T} & L_{22}^{\prime} & L_{23}^{\prime} \\
0 & 0 & 0 \\
0 & 0 & 0
\end{array}\right]
$$

$Q$ is positive semi-definite if:

$$
\begin{aligned}
X^{T} Q X \geq 0 \quad X \in \Re^{6 \times 1} \\
X^{T} Q X=\frac{1}{R_{1} \Delta} X^{T}\left[\begin{array}{ccc}
L_{12}^{\prime T} & L_{22}^{\prime} & L_{23}^{\prime} \\
0 & 0 & 0 \\
0 & 0 & 0
\end{array}\right] X \\
=\frac{1}{R_{1} \Delta} X^{T} \underbrace{\left[\begin{array}{ccc}
L_{12}^{\prime T} & \frac{1}{2} L_{22}^{\prime} & \frac{1}{2} L_{23}^{\prime} \\
\frac{1}{2} L_{22}^{\prime T} & 0 & 0 \\
\frac{1}{2} L_{23}^{\prime T} & 0 & 0
\end{array}\right]}_{\triangleq Q^{\prime}} X
\end{aligned}
$$

In order to prove that $Q$ is positive semi-definite (since $\Delta<0$ ), it's enough to prove that $Q^{\prime}$ is negative semi-definite.

$$
Q^{\prime}=\left[\begin{array}{ccc}
-L_{m G} L_{r M} e^{J n_{G} \theta_{G}} & \frac{1}{2} L_{r G} L_{r M} I_{2} & \frac{1}{2} L_{r G} L_{m M} e^{J n_{M} \theta_{M}} \\
\frac{1}{2} L_{r G} L_{r M} I_{2} & 0 & 0 \\
\frac{1}{2} L_{r G} L_{m M} e^{-J n_{M} \theta_{M}} & 0 & 0
\end{array}\right]
$$

We can see that all the sub-determinant of $Q^{\prime}$ are negative, hence the exponential stability of the $\mathrm{PBC}+\mathrm{P}$ controller is proven. 


\subsection{PBC + PI}

The PBC + PI controller is given by the equation below:

$$
B v_{r G}=\dot{\lambda}^{d}+R L^{-1}(\theta) \lambda^{d}+B\left(K_{p}\left(i_{s G}-i_{s G}^{d}\right)+K_{i} \int\left(i_{s G}-i_{s G}^{d}\right)\right)
$$

where $K_{p}$ and $K_{i}$ are the proportional and integral positive gains. We have:

$$
\begin{aligned}
\int\left(i_{s G}-i_{s G}^{d}\right) & =\frac{-1}{R_{2}}\left(\lambda_{s G M}-\lambda_{s G M}^{d}\right) \\
& =\frac{-1}{R_{2}} \underbrace{\left[\begin{array}{lll}
0 & I_{2} & 0
\end{array}\right]}_{P}(\underbrace{\lambda-\lambda^{d}}_{\tilde{\lambda}})
\end{aligned}
$$

Then,

$$
B v_{r G}=\dot{\lambda}^{d}+R L^{-1}(\theta) \lambda^{d}+K_{p} B P L^{-1}(\theta) \tilde{\lambda}-\frac{K_{i}}{R_{2}} B P \tilde{\lambda}
$$

The closed loop error dynamic is:

$$
\dot{\tilde{\lambda}}=-R L^{-1}(\theta) \tilde{\lambda}-K_{p} B P L^{-1}(\theta) \tilde{\lambda}+\frac{K_{i}}{R_{2}} B P \tilde{\lambda}
$$

Consider the desired energy function given by (33), it's derivative along the trajectories of (50) is:

$$
\begin{aligned}
\dot{V} & =\tilde{\lambda}^{T} R^{-1} \dot{\tilde{\lambda}} \\
& =-\tilde{\lambda}^{T} L^{-1}(\theta) \tilde{\lambda}-K_{p} \tilde{\lambda}^{T} R^{-1} B P L^{-1}(\theta) \tilde{\lambda}+\frac{K_{i}}{R_{2}} \tilde{\lambda}^{T} R^{-1} B P \tilde{\lambda} \\
& =-\tilde{\lambda}^{T} \underbrace{L^{-1}(\theta)}_{>0} \tilde{\lambda}+\tilde{\lambda}^{T} \underbrace{\left[-K_{p} R^{-1} B P L^{-1}(\theta)+\frac{K_{i}}{R_{2}} R^{-1} B P\right]}_{\triangleq M} \tilde{\lambda}
\end{aligned}
$$

To show that the error dynamic (50) is stable, it's enough to prove that $M$ is a negative semi-definite matrix:

$$
M=\frac{1}{R_{1}}\left[\begin{array}{ccc}
-\frac{K_{p}}{|\Delta|} L_{m G} L_{r M} e^{J n_{G} \theta_{G}} & {\left[\frac{K_{p}}{|\Delta|} L_{r G} L_{r M}+\frac{K_{i}}{R_{2}}\right] I_{2}} & \frac{K_{p}}{|\Delta|} L_{r G} L_{m M} e^{J n_{M} \theta_{M}} \\
0 & 0 & 0 \\
0 & 0 & 0
\end{array}\right]
$$

$M$ is a negative semi-definite matrix if: $X^{T} M X \leq 0 \quad X \in \Re^{6 \times 1}$

$$
X^{T} M X=\frac{1}{R_{1}} X^{T} \underbrace{\left[\begin{array}{ccc}
-\frac{K_{p}}{|\Delta|} L_{m G} L_{r M} e^{J n_{G} \theta_{G}} & {\left[\frac{K_{p}}{2|\Delta|} L_{r G} L_{r M}+\frac{K_{i}}{2 R_{2}}\right] I_{2}} & \frac{K_{p}}{2|\Delta|} L_{r G} L_{m M} e^{J n_{M} \theta_{M}} \\
{\left[\frac{K_{p}}{2|\Delta|} L_{r G} L_{r M}+\frac{K_{i}}{2 R_{2}}\right] I_{2}} & 0 & 0 \\
\frac{K_{p}}{2|\Delta|} L_{r G} L_{m M} e^{-J n_{M} \theta_{M}} & 0 & 0
\end{array}\right] X}_{\triangleq M^{\prime}}
$$

To prove that $M$ is a negative semi-definite matrix, it's enough to prove that $M^{\prime}$ is a negative semi-definite matrix, the calculus of the sub-determinants of this latter show that $M^{\prime}$ is a negative semi-definite matrix.

Hence the exponential stability of the PBC + PI controller is proven. 


\section{The construction for BDFTIG}

To establish the complete mathematical representation of the dynamic behaviour of the BDFTIG it is first necessary to clarify the kind of the electromechanical interconnection that exists between the cascaded machines. One of the simplest ways to connect these two machines is in the back-to-back method with no phase inversion on the rotor side, as shown by Figure 6.

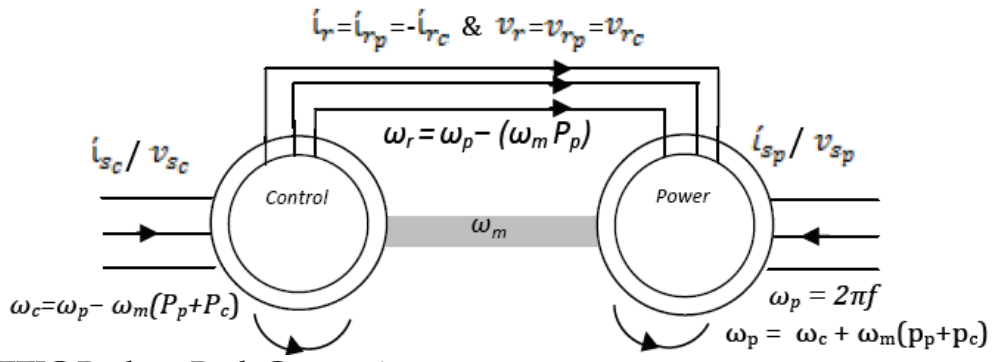

Fig. 6. BDFTIG Back-to-Back Connection

By this connection, the rotor currents produced by the two machines join in the subtractive style, and the rotor voltages have the same signs, i.e. $I_{r p}=-I_{r c}$ and $V_{r p}=V_{r c}$. The chosen connection really affects the distribution of the magnetic fields and flux inside the BDFTIG, producing the two counter-rotating torques as will be discussed in the following sections.

\subsection{Equivalent circuit analysis of the BDFTIG}

Figure 7- shows the equivalent circuit of the BDFTIG from which the electrical system equations can be derived.

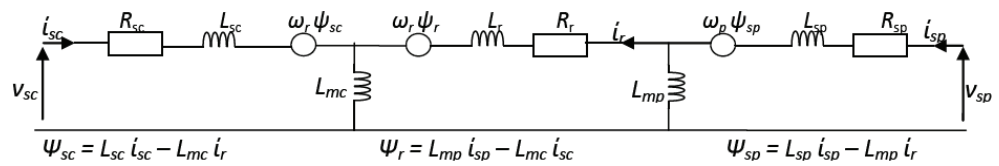

Fig. 7. Equivalent Circuit of the BDFTIG

To simplify the controller algorithm, the machine quantities should be expressed in the d-q frame by employing Park's and Clark's transformation. The reason of this transformations is to remove as many time-varying quantities from the system as possible. By converting the three-phase machine to its two-phase equivalent and selecting the suitable reference frame, all the time-varying inductances in both the stator and the rotor are eliminated, allowing for a simple however complete dynamic model of the electric machine. From these equivalent circuits the electrical equations of BDFTIG can be determined as shown in the next section.

\subsection{Electrical system equations for BDFTIG}

Starting with the power machine, the general form of the vector equations of the BDFTIG can be written as:

$$
\begin{aligned}
v_{s p}^{q} & =R_{s p} i_{s p}^{q}+L_{s p} \frac{d i_{s p}^{q}}{d t}+\omega_{p} L_{s p} i_{s p}^{d}+L_{m p} \frac{d i_{r p}^{q}}{d t}+\omega_{p} L_{m p} i_{r p}^{d} \\
v_{s p}^{q} & =R_{s p} i_{s p}^{q}+\left(L_{s p} i_{s p}^{q}+L_{m p} i_{r p}^{q}\right) s+\left(L_{s p} i_{s p}^{d}+L_{m p} i_{r p}^{d}\right) \omega_{p}
\end{aligned}
$$




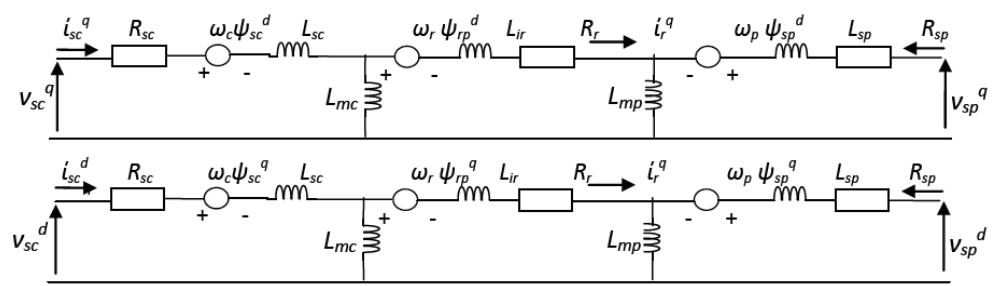

Fig. 8. Equivalent Circuits of d-q BDFTIG

The flux linkage current relations are:

$$
\begin{aligned}
\Psi_{s p}^{q} & =L_{s p} i_{s p}^{q}+L_{m p} i_{r p}^{q} \\
\Psi_{s p}^{d} & =L_{s p} i_{s p}^{d}+L_{m p} i_{r p}^{d} \\
v_{s p}^{q} & =R_{s p} i_{s p}^{q}+\frac{d \Psi_{s p}^{q}}{d t}+\omega_{p} \Psi_{s p}^{d} \\
v_{r p}^{q} & =R_{r p} i_{r p}^{q}+L_{r p} \frac{d i_{r p}^{q}}{d t}+\omega_{r} L_{r p} i_{r p}^{d}+L_{m p} \frac{d i_{s p}^{q}}{d t}+\omega_{r} L_{m p} i_{s p}^{d} \\
v_{r p}^{q} & =R_{r p} i_{r p}^{q}+\left(L_{r p} i_{r p}^{q}+L_{m p} i_{s p}^{q}\right) s+\left(L_{r p} i_{r p}^{d}+L_{m p} i_{s p}^{d}\right) \omega_{r}
\end{aligned}
$$

We have also:

$$
\begin{aligned}
\Psi_{r p}^{q} & =L_{r p} i_{r p}^{q}+L_{m p} i_{s p}^{q} \\
\Psi_{r p}^{d} & =L_{r p} i_{r p}^{d}+L_{m p} i_{s p}^{d} \\
v_{r p}^{q} & =R_{r p} i_{s p}^{q}+\frac{d \Psi_{r p}^{q}}{d t}+\omega_{p} \Psi_{r p}^{d} \\
v_{s p}^{d} & =R_{s p} i_{s p}^{d}+L_{s p} \frac{d i_{s p}^{d}}{d t}-\omega_{p} L_{s p} i_{s p}^{q}+L_{m p} \frac{d i_{r p}^{d}}{d t}-\omega_{p} L_{m p} i_{r p}^{q} \\
v_{s p}^{d} & =R_{s p} i_{s p}^{d}+\left(L_{s p} i_{s p}^{d}+L_{m p} i_{r p}^{d}\right) s-\left(L_{s p} i_{s p}^{q}+L_{m p} i_{r p}^{q}\right) \omega_{p} \\
v_{s p}^{d} & =R_{s p} i_{s p}^{d}+\frac{d \Psi_{s p}^{d}}{d t}-\omega_{p} \Psi_{s p}^{q} \\
v_{r p}^{d} & =R_{r p} i_{r p}^{d}+L_{r p} \frac{d i_{r p}^{d}}{d t}+\omega_{r} L_{r p} i_{r p}^{q}+L_{m p} \frac{d i_{s p}^{d}}{d t}+\omega_{r} L_{m p} i_{s p}^{q} \\
v_{r p}^{d} & =R_{r p} i_{r p}^{d}+\left(L_{r p} i_{r p}^{d}+L_{m p} i_{s p}^{d}\right) s+\left(L_{r p} i_{r p}^{q}+L_{m p} i_{s p}^{q}\right) \omega_{r} \\
v_{r p}^{d} & =R_{r p} i_{s p}^{d}+\frac{d \Psi_{r p}^{d}}{d t}+\omega_{p} \Psi_{r p}^{q}
\end{aligned}
$$

Electrical system equations for control machine:

$$
\begin{aligned}
v_{s c}^{q} & =R_{s c} i_{s c}^{q}+L_{s c} \frac{d i_{s c}^{q}}{d t}+\omega_{c} L_{s c} i_{s c}^{d}+L_{m c} \frac{d i_{r c}^{q}}{d t}+\omega_{c} L_{m c} i_{r c}^{d} \\
v_{s c}^{q} & =R_{s c} i_{s c}^{q}+\left(L_{s c} i_{s c}^{q}+L_{m c} i_{r c}^{q}\right) s+\left(L_{s c} i_{s p}^{d}+L_{m c} i_{r c}^{d}\right) \omega_{c}
\end{aligned}
$$


The flux linkage current relations are:

$$
\begin{aligned}
\Psi_{r c}^{q} & =L_{s c} i_{s c}^{q}+L_{m c} i_{r c}^{q} \\
\Psi_{s c}^{d} & =L_{s c} i_{s p}^{d}+L_{m c} i_{r c}^{d} \\
v_{s c}^{q} & =R_{s c} i_{s c}^{q}+\frac{d \Psi_{s c}^{q}}{d t}+\omega_{c} \Psi_{s c}^{d} \\
v_{r c}^{q} & =R_{r c} i_{r c}^{q}+L_{r c} \frac{d i_{r c}^{q}}{d t}+\omega_{r} L_{r c} i_{r c}^{d}+L_{m c} \frac{d i_{s c}^{q}}{d t}+\omega_{r} L_{m c} i_{s c}^{d} \\
v_{r c}^{q} & =R_{r c} i_{r c}^{q}+\left(L_{r c} i_{r c}^{q}+L_{m c} i_{s c}^{q}\right) s+\left(L_{r p} i_{r p}^{d}+L_{m c} i_{s c}^{d}\right) \omega_{r}
\end{aligned}
$$

and:

$$
\begin{aligned}
\Psi_{r c}^{q} & =L_{r c} i_{r c}^{q}+L_{m c} i_{s c}^{q} \\
\Psi_{r c}^{d} & =L_{r c} i_{r c}^{d}+L_{m c} i_{s c}^{d} \\
v_{r c}^{q} & =R_{r c} i_{r c}^{q}+\frac{d \Psi_{r c}^{q}}{d t}+\omega_{r} \Psi_{r c}^{d} \\
v_{s c}^{d} & =R_{r c} i_{s c}^{d}+L_{s c} \frac{d i_{s c}^{d}}{d t}-\omega_{c} L_{s c} i_{s c}^{q}+L_{m c} \frac{d i_{r c}^{d}}{d t}-\omega_{c} L_{m c} i_{r c}^{q} \\
v_{s c}^{d} & =R_{s c} i_{s c}^{d}+\left(L_{s c} i_{s c}^{d}+L_{m c} i_{r c}^{d}\right) s-\left(L_{s c} i_{s c}^{q}+L_{m c} i_{r c}^{q}\right) \omega_{c} \\
v_{s c}^{d} & =R_{s c} i_{s c}^{d}+\frac{d \Psi_{s c}^{d}}{d t}-\omega_{c} \Psi_{s c}^{q} \\
v_{r c}^{d} & =R_{r c} i_{r c}^{d}+L_{r c} \frac{d i_{r c}^{d}}{d t}+\omega_{c} L_{r c} i_{r c}^{q}+L_{m c} \frac{d i_{s c}^{d}}{d t}+\omega_{r} L_{m c} i_{s c}^{q} \\
v_{r c}^{d} & =R_{r c} i_{r c}^{d}+\left(L_{r c} i_{r c}^{d}+L_{m c} i_{s c}^{d}\right) s+\left(L_{r p} i_{r p}^{q}+L_{m c} i_{s c}^{q}\right) \omega_{r} \\
v_{r c}^{d} & =R_{r c} i_{r c}^{d}+\frac{d \Psi_{r c}^{d}}{d t}+\omega_{r} \Psi_{r c}^{q}
\end{aligned}
$$

As mentioned before, for the BDFTIG with the back-to-back configuration and with no phase inversion, the rotor currents of the individual machines have the opposite signs, the fluxes inside the rotor combine to produce the essential rotor flux, hence; $i_{r p}=-i_{r c}=i_{r}, \Psi_{r}=$ $\Psi_{r p}-\Psi_{r c}, v_{r p}=v_{r c}, 0=v_{r p}-v_{r c}$

$$
\begin{aligned}
0_{r}^{q} & =R_{r p} i_{r}^{q}+L_{r p} \frac{d i_{r}^{q}}{d t}+\omega_{r} L_{r p} i_{r}^{d}+L_{m p} \frac{d i_{s p}^{q}}{d t}+\omega_{r} L_{m p} i_{s p}^{d}+R_{r c} i_{r}^{q}+L_{r c} \frac{d i_{r}^{q}}{d t}+\omega_{r} L_{r c} i_{r}^{d} \\
& -L_{m c} \frac{d i_{s c}^{q}}{d t}-\omega_{r} L_{m c} i_{s c}^{d}
\end{aligned}
$$

But $L_{r}=L_{r p}+L_{r c}$ and $R_{r}=R_{r p}+R_{r c}$

$$
\begin{aligned}
& 0_{r}^{q}=R_{r} i_{r}^{q}+L_{r} \frac{d i_{r}^{q}}{d t}+\omega_{r} L_{r} i_{r}^{d}+L_{m p} \frac{d i_{s p}^{q}}{d t}+\omega_{r} L_{m p} i_{s p}^{d}-L_{m c} \frac{d i_{s c}^{q}}{d t}-\omega_{r} L_{m c} i_{s c}^{d} \\
& 0_{r}^{q}=R_{r} i_{r}^{q}+\left(L_{r} i_{r}^{q}+L_{m p} i_{s p}^{q}-L_{m c} i_{s c}^{q}\right) s+\left(L_{r} i_{r}^{d}+L_{m p} i_{s p}^{d}-L_{m c} i_{s c}^{d}\right) \omega_{r}
\end{aligned}
$$


The flux linkage current relations are:

$$
\begin{aligned}
\Psi_{r}^{q} & =L_{r} i_{r}^{q}+L_{m p} i_{s p}^{q}-L_{m c} i_{s c}^{q} \\
\Psi_{r}^{d} & =L_{r} i_{r}^{d}+L_{m p} i_{s p}^{d}-L_{m c} i_{s c}^{d} \\
0_{r}^{q} & =R_{r} i_{r}^{q}+\frac{d \Psi_{r}^{q}}{d t}+\omega_{r} \Psi_{r}^{d} \\
0_{r}^{q} & =R_{r} i_{r}^{d}+L_{r} \frac{d i_{r}^{d}}{d t}+\omega_{r} L_{r} i_{r}^{q}+L_{m p} \frac{d i_{s p}^{d}}{d t}+\omega_{r} L_{m p} i_{s p}^{q}-L_{m c} \frac{d i_{s c}^{d}}{d t}-\omega_{r} L_{m c} i_{s c}^{q} \\
0_{r}^{q} & =R_{r} i_{r}^{d}+\left(L_{r} i_{r}^{d}+L_{m p} i_{s p}^{d}-L_{m c} i_{s c}^{d}\right) s+\left(L_{r} i_{r}^{q}+L_{m p} i_{s p}^{q}-L_{m c} i_{s c}^{q}\right) \omega_{r} \\
0_{r}^{q} & =R_{r} i_{r}^{d}+\frac{d \Psi_{r}^{d}}{d t}+\omega_{r} \Psi_{r}^{q}
\end{aligned}
$$

The electrical torque creation in the power machine is governed by the same principles that apply to any induction machine. The general equation of the electrical torque in this case is simply:

$$
T_{e}=\frac{3}{2}\left(\frac{P}{2}\right) \Psi_{m} I_{r}
$$

In the $\mathrm{d}$-q reference frame, however, the last equation is rearranged to show the torque as a function of certain control parameter. As the power machine is grid connected, it will have a constant voltage. The torque could be:

$$
T_{p}=\frac{3}{2}\left(\frac{P_{p}}{2}\right)\left(\Psi_{s p}^{q} i_{s p}^{d}-\Psi_{s p}^{d} i_{s p}^{q}\right)
$$

It is clear from the above equation that the only control variables are the $\mathrm{d}$-q components of the stator current, because the power machine stator fluxes are almost constant. Furthermore, when the controller reference frame is aligned with one of the flux components, the number of the control variables is reduced. To derive the electrical torque for the control machine, we can use the same general equation for the electrical torque. This case cannot be simplified because the stator fluxes of the control machine will be variable. The control machine torque must be expressed as a function of the excitation current and the purpose in this research is to provide a flexible power control of the BDFIG. So the next equation is the control machine torque, and it is given in terms of the future control quantities.

$$
T_{c}=-\frac{3}{2}\left(\frac{P_{c}}{2}\right) L_{m c}\left(i_{s c}^{d} i_{r}^{q}-i_{s c}^{q} i_{r}^{d}\right)
$$

The option of the rotor current as the second variable is clearly shown and that there exists an electric coupling between the two stators of the BDFTIG, which is achieved through the common rotor current. This reflects the behaviour of the inner workings of the BDFTIG. The total electric torque (Te) for the BDFTIG is the sum of the individual electrical torques of both machines:

$$
T_{e}=-\frac{3}{4}\left[P_{p}\left(\Psi_{s p}^{q} i_{s p}^{d}-\Psi_{s p}^{d} i_{s p}^{q}\right)+P_{c} L_{m c}\left(i_{s c}^{d} i_{r}^{q}-i_{s c}^{q} i_{r}^{d}\right)\right]
$$

The electric torque equation is defined by the friction and total inertia of the power and control machines:

$$
T_{e}=T_{L}+\left(B_{F}^{p}+B_{F}^{c}\right) \omega_{m}+\left(j_{s}^{p}+j_{s}^{c}\right) \frac{d \omega_{m}}{d t}
$$


Rearranging the last equation to derive the shaft speed:

$$
\frac{d \omega_{m}}{d t}=\frac{1}{j_{s}^{p}+j_{s}^{c}}\left[T_{e}-T_{L}-\left(B_{F}^{p}+B_{F}^{c}\right) \omega_{m}\right]
$$

Hence, the shaft speed is

$$
\omega_{m}=\frac{T_{e}-T_{L}}{\left(B_{F}^{p}+B_{F}^{c}\right)+\left(j_{s}^{p}+j_{s}^{c}\right) s}
$$

\subsection{Simulation of the BDFTIG Model}

The BDFTIG model was tested to determine if it was a true representation of the actual generator. Using Matlab/Simulink to test the BDFTIG, the main tests consisted of disabling one side of the BDFTIG and applying a constant AC voltage on the opposite side, at the same time as changing the load torque to allow both motoring and generation modes of operation. The short circuit test consisted of shorting the stator side of the control machine, and a natural speed of $900 \mathrm{rpm}$ was recorded, because both machines have four poles each, as shown in Figure 9. For the next test, the load torque was decreased at time 2.25 s to put the BDFTIG into the generation mode as shown in Figure 10. The system responded as expected by increasing its speed and moving into the super-synchronous mode of operation, the electrical torque changed at the same time as the load demand. In this section, the dynamic model of the generator was developed based on the selected $d-q$ reference frame. The model was implemented and tested in MATLAB/Simulink. The simulation results verified that the model can correctly describe the dynamic behaviour of BDFTIG design.

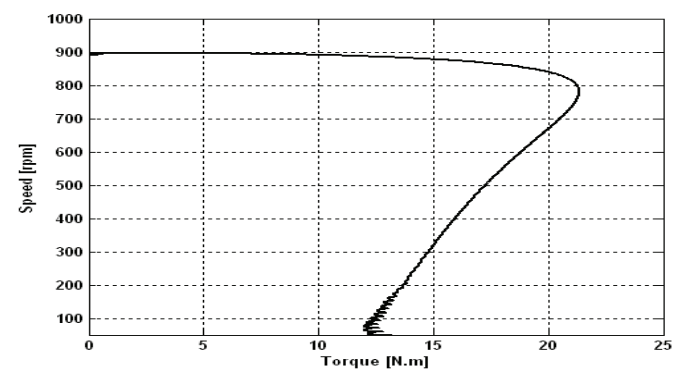

Fig. 9. Speed-Torque Curve of BDFTIG with short circuit test.

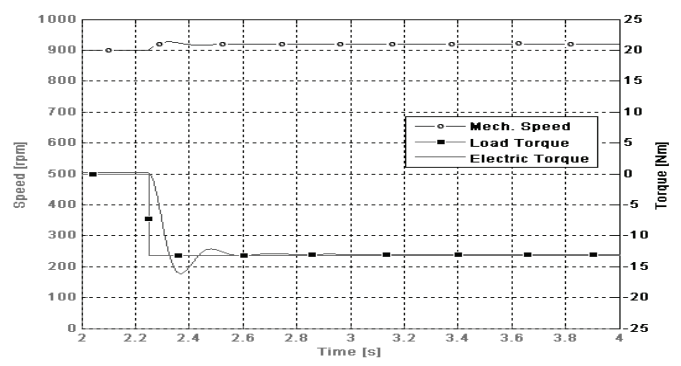

Fig. 10. Generation Mode of BDFTIG. 
In this section, the dynamic model of the generator was developed based on the selected $\mathrm{d}-\mathrm{q}$ reference frame. The model was implemented and tested in MATLAB/Simulink. The simulation results verified that the model can correctly describe the dynamic behaviour of BDFTIG design.

\section{Experimentation}

In order to validate the new controllers, experiments were conducted on a real system. The following controllers were implemented: $\mathrm{PBC}, \mathrm{PBC}+$ Proportional action on stator currents, PI controller on stator currents, and a combination of PBC and PI control. The experiments were done in the IRII-UPC (Institute of Robotics and Industrial Informatics - University Polytechnic of Catalonia) where a $200 \mathrm{~W}$ DFIG interconnected with an IM prototype is available (see Fig. (11)). The setup was controlled using a computer working under RT-Linux operating system. With the PBC, only the position sensors of the Generator and the Induction machine were used for the control. For the Proportional and PI controllers of the electrical subsystem, measurement of the two stator currents were also needed. In order to show the behaviour of the system under different load conditions, a non-measured load torque was applied.

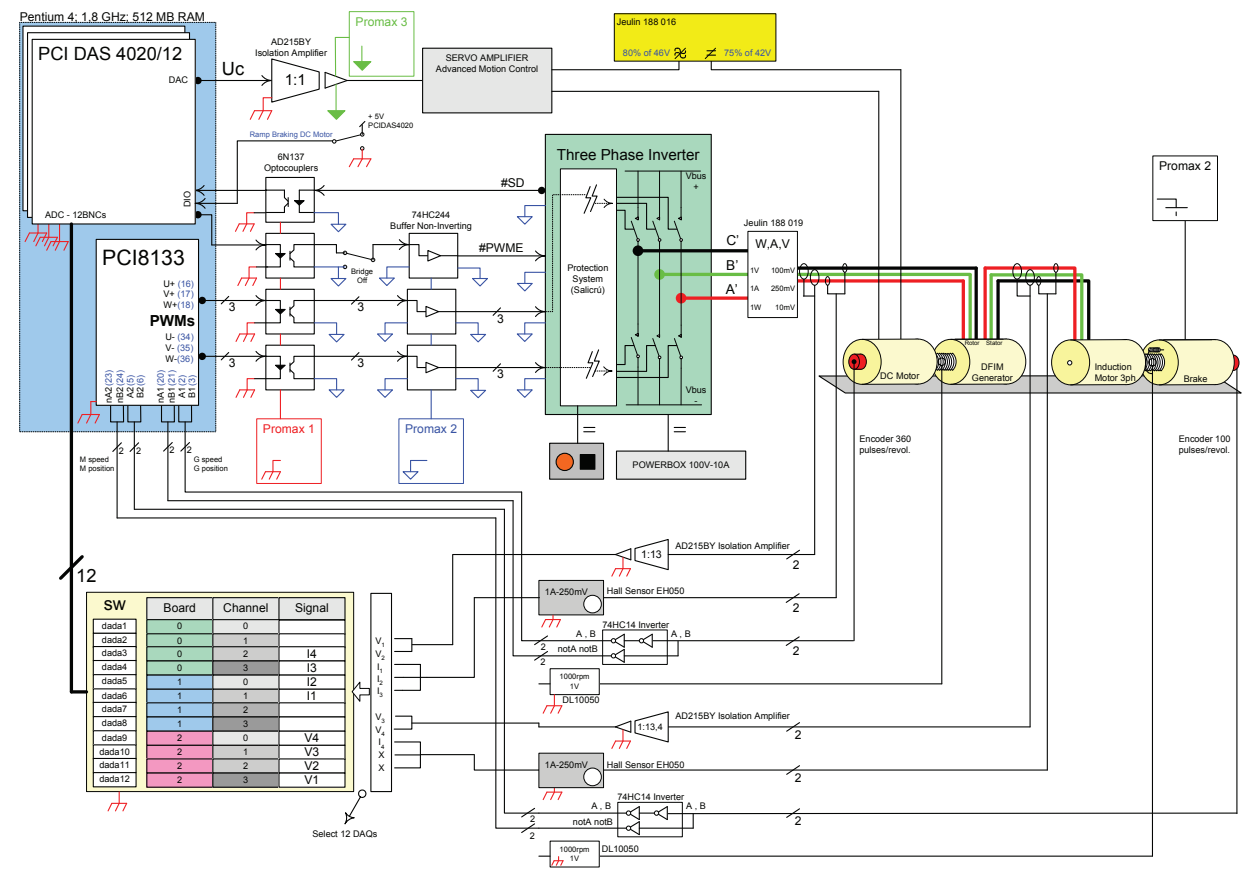

Fig. 11. Experimental setup

Since a load torque sensor was not available for the acquisition, we built an estimator of the resistive torque based on the measurement of the mechanical IM speed. 


\subsection{Estimation of the load torque}

The mechanical dynamics of the IM is given by:

$$
J_{M} \ddot{\theta}_{M}=\tau_{M}-\tau_{L M}-B_{M} \dot{\theta}_{M}
$$

Since the asymptotic stability of the electrical subsystem $\Sigma_{e}$ is proven we can consider that in the steady state $\tau_{M} \rightarrow \tau_{M}^{d}$ (exponentially). Then,we have in the steady state the following:

$$
J_{M} \ddot{\theta}_{M}=\tau_{M}^{d}-\underbrace{\tau_{L M}-B_{M} \dot{\theta}_{M}}_{\tau_{M L}}
$$

Hence, a linear load torque observer was designed (with $l_{1}, l_{2}$ are design parameters):

$$
\begin{aligned}
\dot{\hat{\omega}}_{m M} & =\left(\tau_{M}^{d}-\hat{\tau}_{M L}\right) / J_{M}+l_{1}\left(\hat{\omega}_{m M}-\omega_{m M}\right) \\
\dot{\hat{\tau}}_{M L} & =l_{2}\left(\hat{\omega}_{m M}-\omega_{m M}\right)
\end{aligned}
$$

\subsection{PBC}
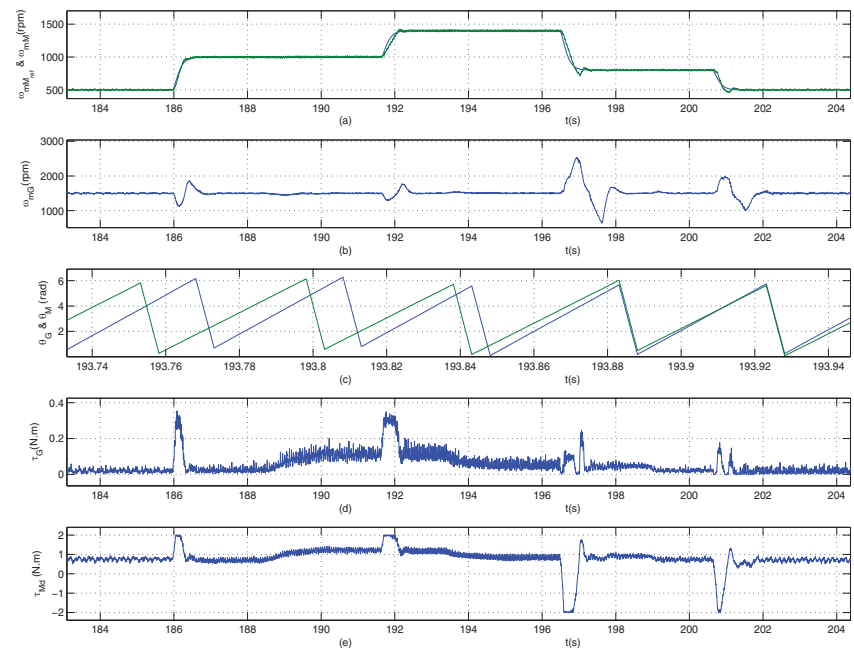

Fig. 12. PBC-(a) Regulated Motor speed and its reference. (b)Generator speed. (c) DFIG \& IM rotor position. (d) Generator torque (e) Motor desired torque.

Figure 12 presents the mechanical IM speed and its smooth reference, the mechanical DFIG speed, the DFIG and IM rotor positions, the DFIG torque $\tau_{G}$ and the IM desired torque $\tau_{M d}$. The real IM speed tracks the reference very well, i.e. low overshoot and no steady state error are observed. Figure 13 shows the stator currents $i_{s a}$ and $i_{s b}$, and their references over a suitable period of time. The stator currents do not track exactly their desired values but are bounded. This is because the goal of the PBC is to track the IM speed and to keep internal signals bounded.

Figure 14 shows the DFIG rotor currents $i_{r G a}$ and $i_{r G b}$, and their references over a period of time. Again, these currents are sinusoidal and bounded.

Figure 15 presents the DFIG rotor voltages $v_{r G a}$ and $v_{r G b}$, the IM rotor speed $\omega_{m M}$ and its estimation $\hat{\omega}_{m M}$, the estimated IM load torque $\hat{\tau}_{M L}$, and the estimated IM speed, given by 

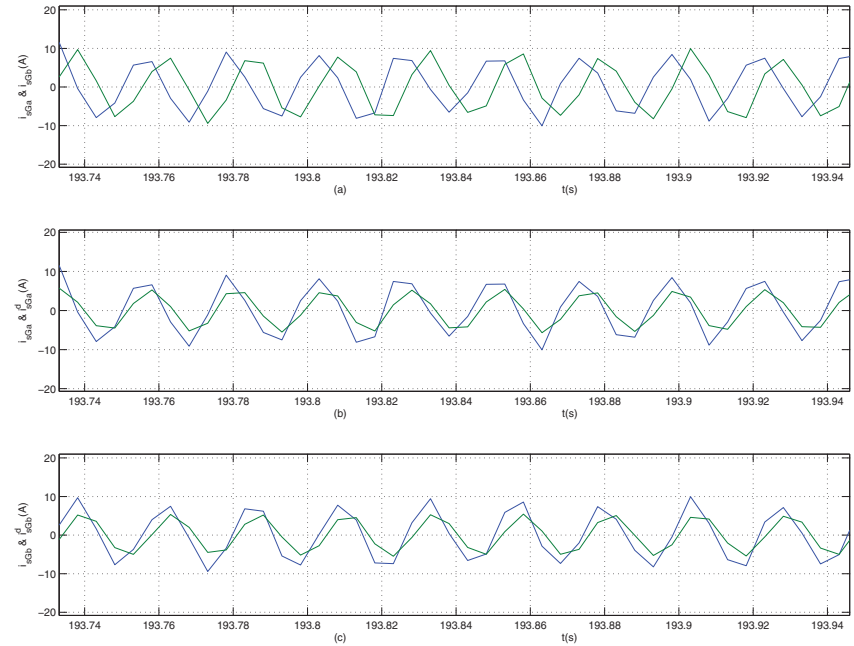

Fig. 13. PBC-(a) $i_{s a}, i_{s b}$ (b) $i_{s a}^{d}, i_{s a}$ (c) $i_{s b}^{d}, i_{s b}$
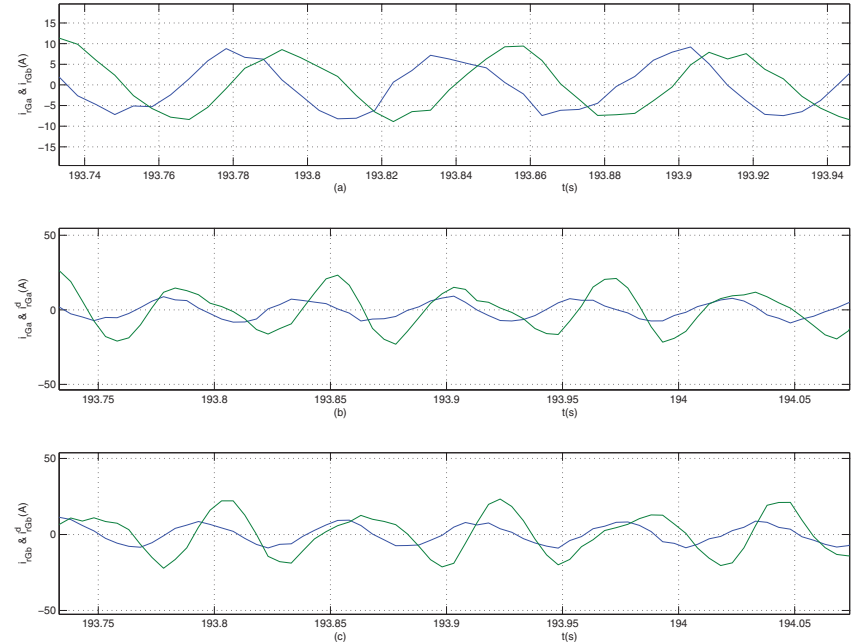

Fig. 14. PBC-(a) $i_{r G a}, i_{r G b}$ (b) $i_{r G a^{\prime}}^{d} i_{r G a}$ (c) $i_{r G b^{\prime}}^{d} i_{r G b}$. 

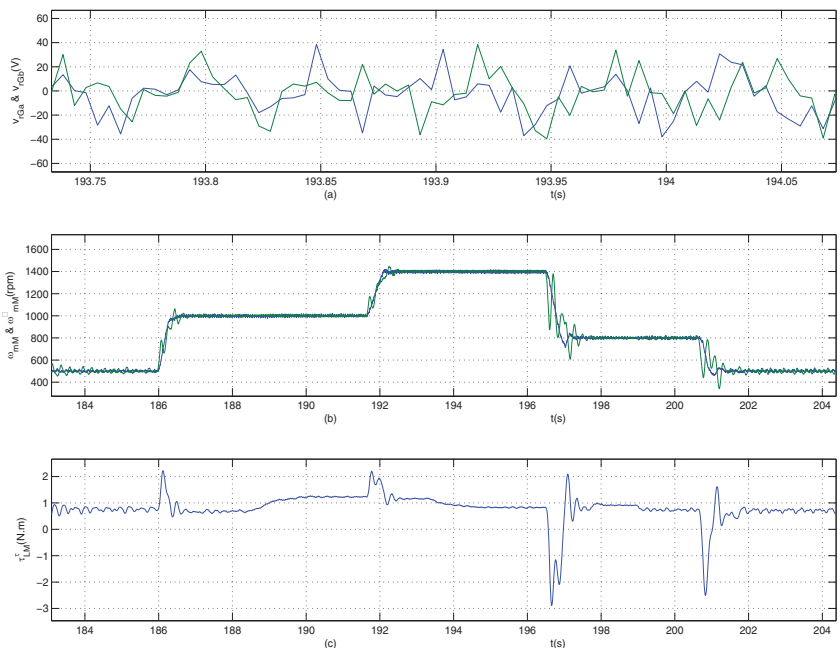

Fig. 15. PBC-(a) $v_{r G a}, v_{r G b}$ (b) $\omega_{m M}, \hat{\omega}_{m M}$ (c) $\hat{\tau}_{M L}$.

(76)-(77), is tracking the real speed. Hence, a good estimation of the real IM load torque is obtained. It has to be noticed that the IM rated torque is $0.7 \mathrm{Nm}$.

It can be concluded that the PBC provides good practical performance even when the applied load torque is twice the magnitude of the nominal load torque of the IM.

\subsection{PBC + P}
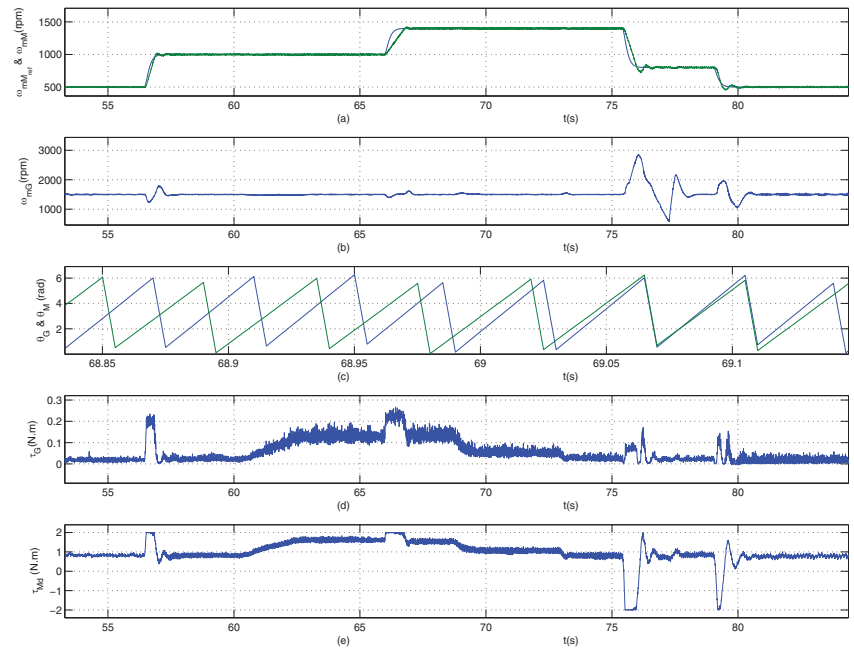

Fig. 16. PBC+P-(a) Regulated Motor speed and its reference. (b)Generator speed. (c) DFIG \& IM rotor position. (d)Generator torque (e) Motor desired torque.

As with the $\mathrm{PBC}$ alone, the results obtained with the $\mathrm{PBC}+\mathrm{P}$ are given in figures 16-19. On the whole, the system behaviour is the same as the PBC alone. One difference that is noticeable is 

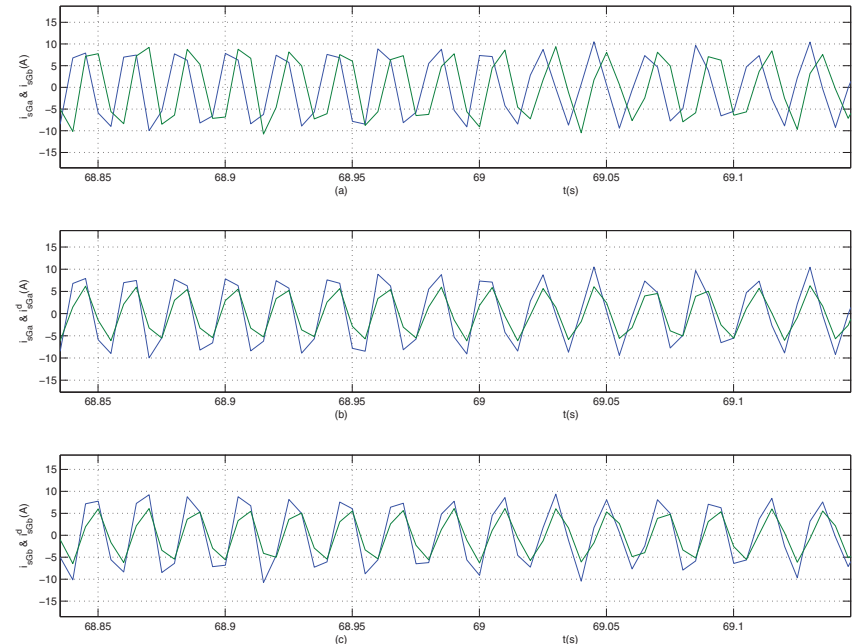

Fig. 17. PBC+P-(a) $i_{s G a}, i_{s G b}$ (b) $i_{s G a^{\prime}}^{d} i_{s G a}$ (c) $i_{s G b^{\prime}}^{d} i_{s G b}$.
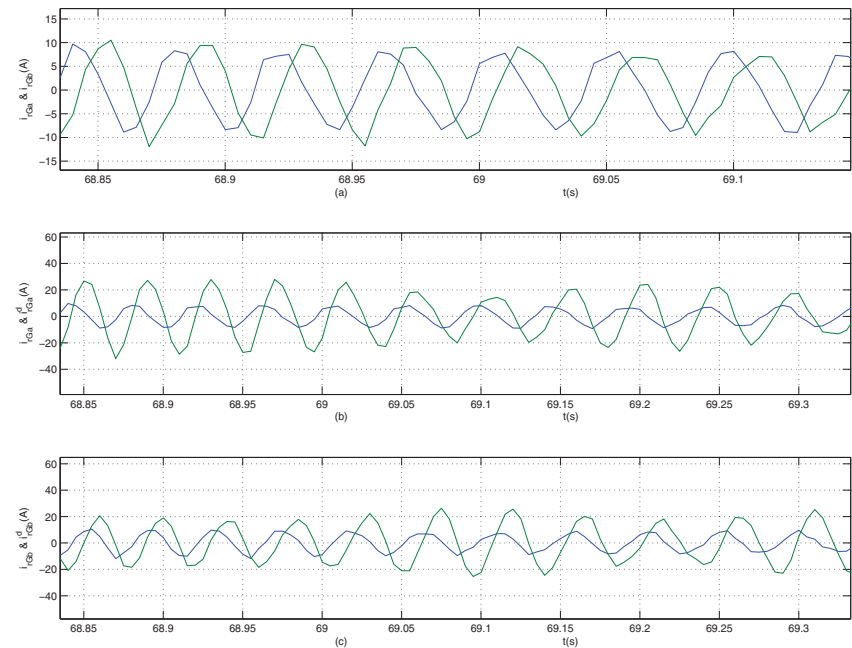

Fig. 18. PBC+P-(a) $i_{r G a}, i_{r G b}$ (b) $i_{r G a^{\prime}}^{d} i_{r G a}$ (c) $i_{r G b^{\prime}}^{d} i_{r G b}$.

the small error between the desired stator currents and the real ones thanks to the proportional controller.

The $\mathrm{PBC}+\mathrm{P}$ controller exhibits good practical performance but not significantly better than those obtained with the PBC alone.

\subsection{PBC + PI}

Again, as for the $\mathrm{PBC}$ and the $\mathrm{PBC}+\mathrm{P}$ controllers, figures 20-23 show the results. It can be seen in figure 21 that the integral actions on the stator currents do not decrease the error significantly between the real and desired values in comparison with the results for the $\mathrm{PBC}+\mathrm{P}$ 

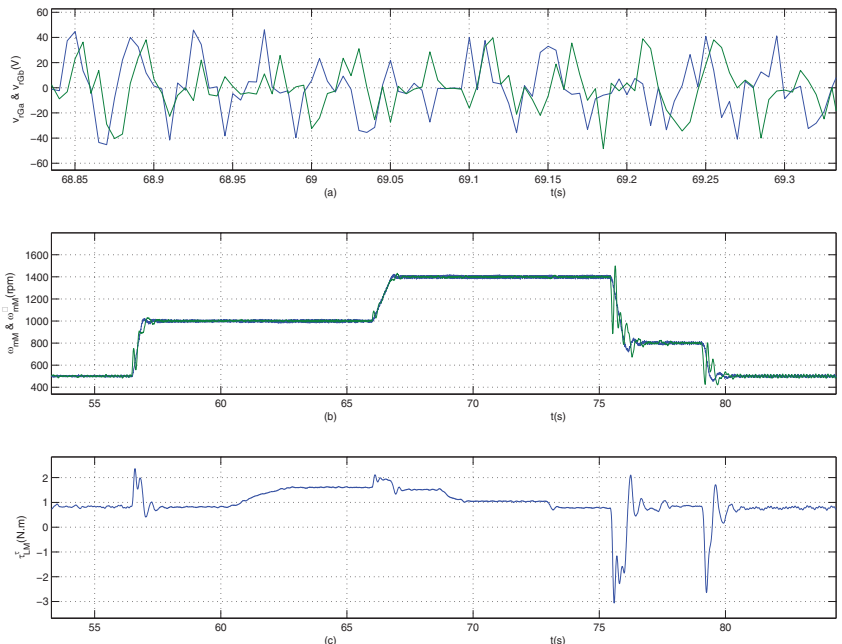

Fig. 19. $\mathrm{PBC}+\mathrm{P}-(\mathrm{a}) v_{r G a}, v_{r G b}$ (b) $\omega_{m M}, \hat{\omega}_{m M}$ (c) $\hat{\tau}_{M L}$.
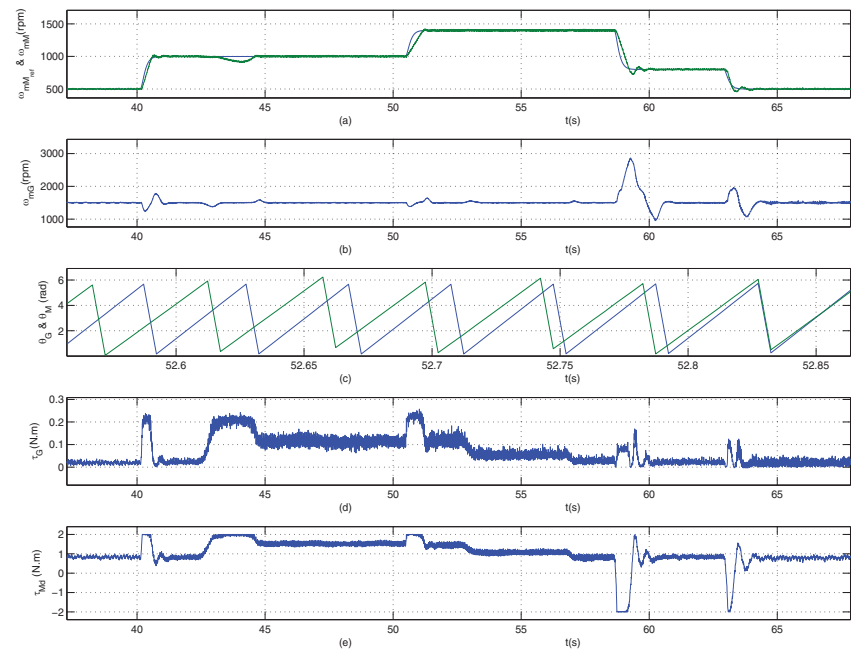

Fig. 20. PBC+PI-(a) Regulated Motor speed and its reference. (b)Generator speed. (c) DFIG \& IM rotor position. (d)Generator torque (e) Motor desired torque. 


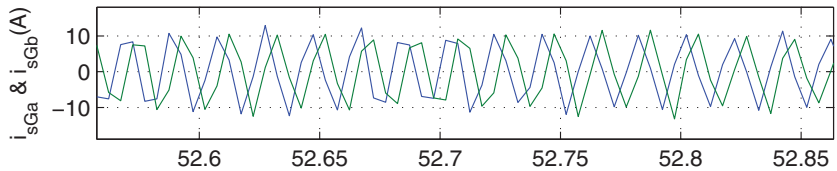

(a)

$\mathrm{t}(\mathrm{s})$

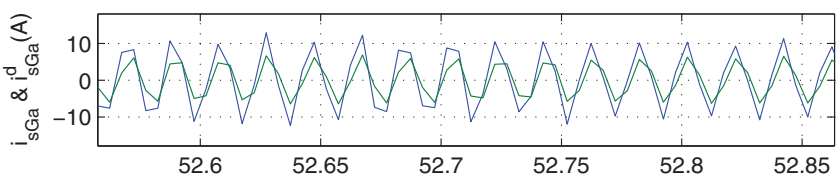

(b)

$\mathrm{t}(\mathrm{s})$

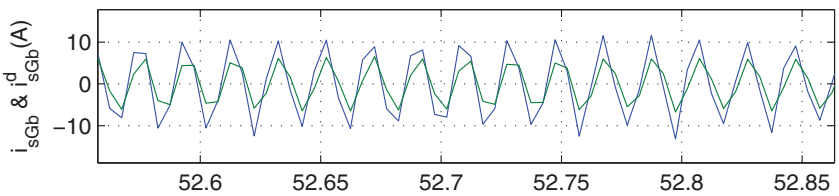

(c)

$t(s)$

Fig. 21. PBC+PI-(a) $i_{s G a}, i_{s G b}$ (b) $i_{s G a^{\prime}}^{d} i_{s G a}$ (c) $i_{s G b^{d}}^{d} i_{s G b}$.

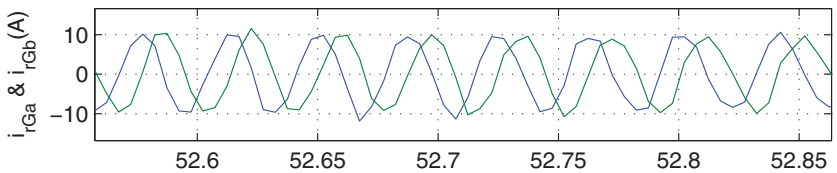

(a)

$\mathrm{t}(\mathrm{s})$

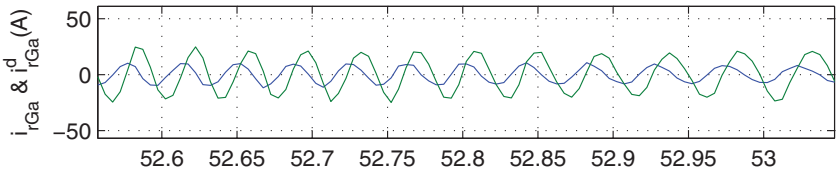

(b)

$\mathrm{t}(\mathrm{s})$

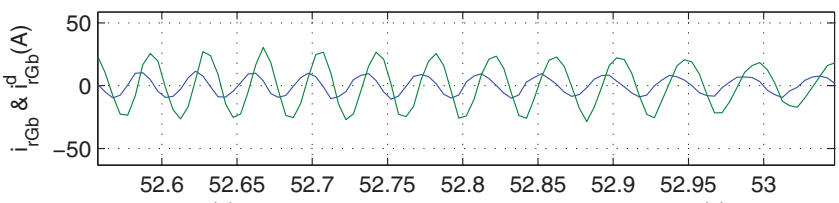

(c)

$\mathrm{t}(\mathrm{s})$

Fig. 22. PBC+PI-(a) $i_{r G a}, i_{r G b}$ (b) $i_{r G a^{\prime}}^{d} i_{r G a}$ (c) $i_{r G b^{\prime}}^{d} i_{r G b}$.

controller (see fig. 17). This is due to the fact that the reference values are sinusoidal and that the bandwidth of the PI controllers cannot be increased sufficiently experimentally.

It can be concluded that the PI action on the stator currents does not improve significantly the performance obtained with the $\mathrm{PBC}+\mathrm{P}$ controller.

\subsection{PI}

The PI control law (with $K_{p}$ and $K_{i}$ are proportional and integral gains) is given below:

$$
B v_{r G}=B\left(K_{p}\left(i_{s G}-i_{s G}^{d}\right)+K_{i}\left(i_{s G}-i_{s G}^{d}\right)\right)
$$




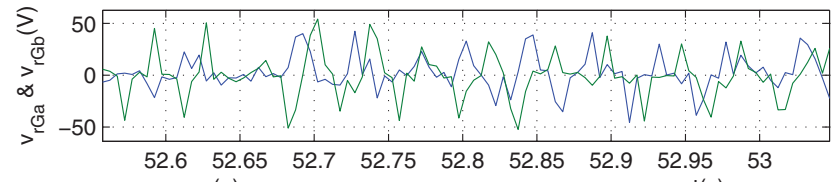

(a)

$\mathrm{t}(\mathrm{s})$

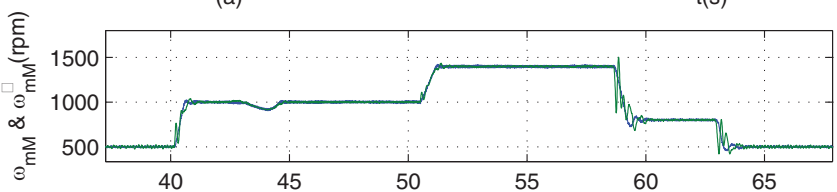

(b)

$\mathrm{t}(\mathrm{s})$

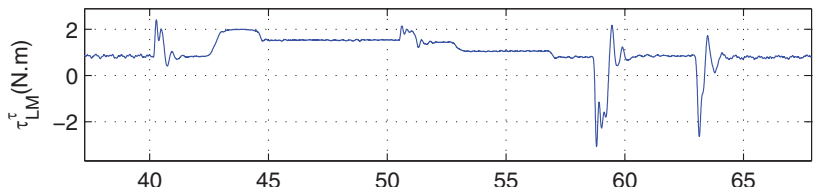

(c)

$\mathrm{t}(\mathrm{s})$

Fig. 23. PBC+PI-(a) $v_{r G a}, v_{r G b}$ (b) $\omega_{m M}, \hat{\omega}_{m M}$ (c) $\hat{\tau}_{M L}$.
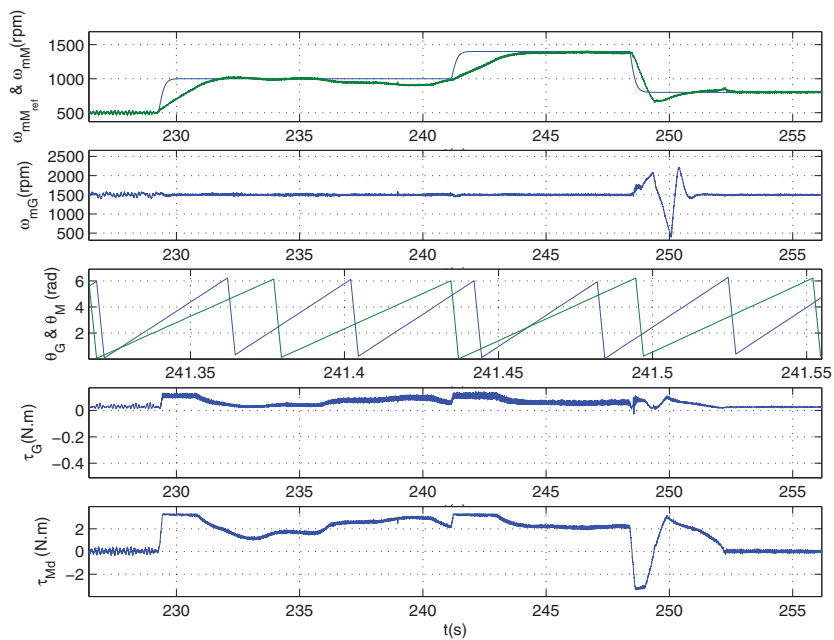

Fig. 24. PI-(a) Regulated Motor speed and its reference. (b)Generator speed. (c) DFIG \& IM rotor position. (d) Generator torque (e) Motor desired torque. 

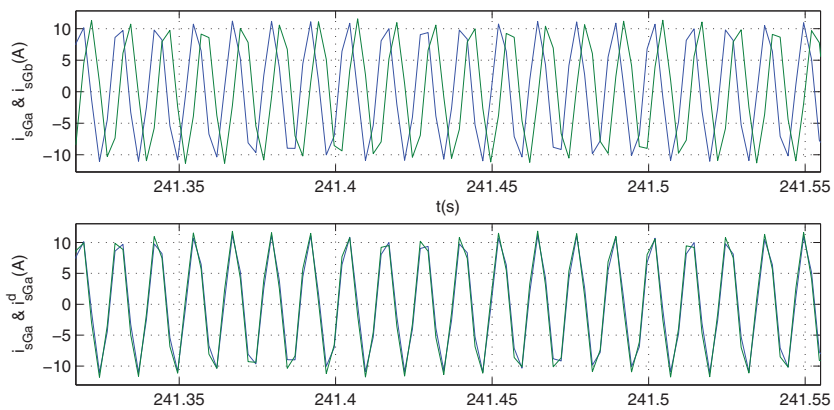

$\mathrm{t}(\mathrm{s})$

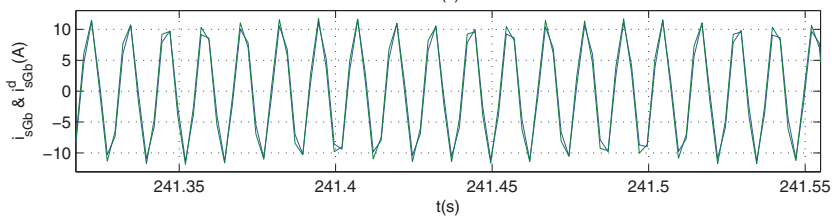

Fig. 25. PI-(a) $i_{s G a}, i_{s G b}$ (b) $i_{s G a^{\prime}}^{d} i_{s G a}$ (c) $i_{s G b}^{d}, i_{s G b}$.
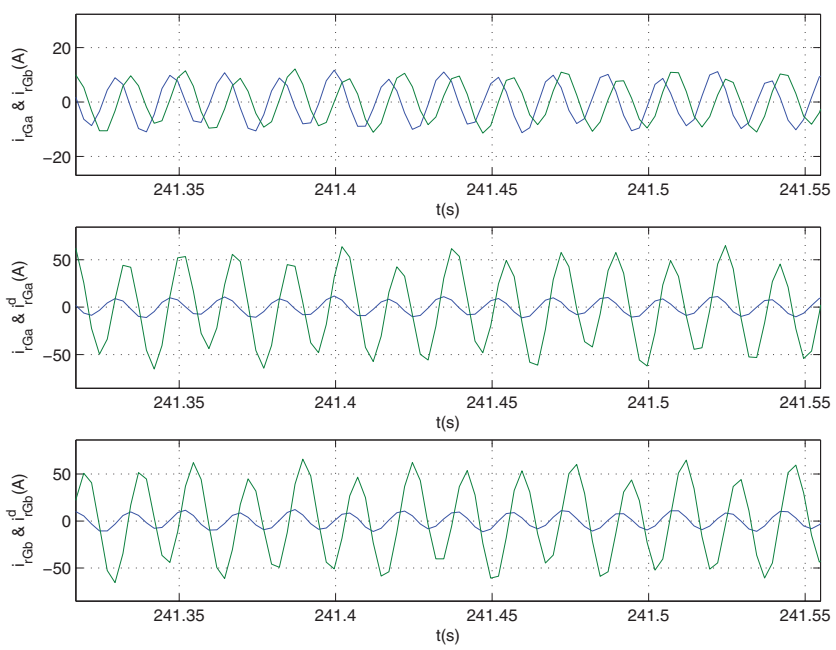

Fig. 26. PI-(a) $i_{r G a}, i_{r G b}$ (b) $i_{r G a}^{d}, i_{r G a}$ (c) $i_{r G b}^{d}, i_{r G b}$.

Finally, in order to obtain a significant comparison between controllers, a PI-based control has been designed without a PBC, i.e. there is one PI controller for each stator current. Figures 24-27 show the results. These results show clearly that the system behaviour is much deteriorated in comparison with the results obtained with the previous controllers. Even if there is no IM speed error in the steady state, the speed does not track its reference during transients, and there is a speed error when a load torque is applied. This is mainly due to the saturation of the desired IM torque at a value four times its nominal value. Consequently, the stator currents are very large, i.e. their magnitude is about twice those currents with the PBC, and so significant stator losses can be expected. 

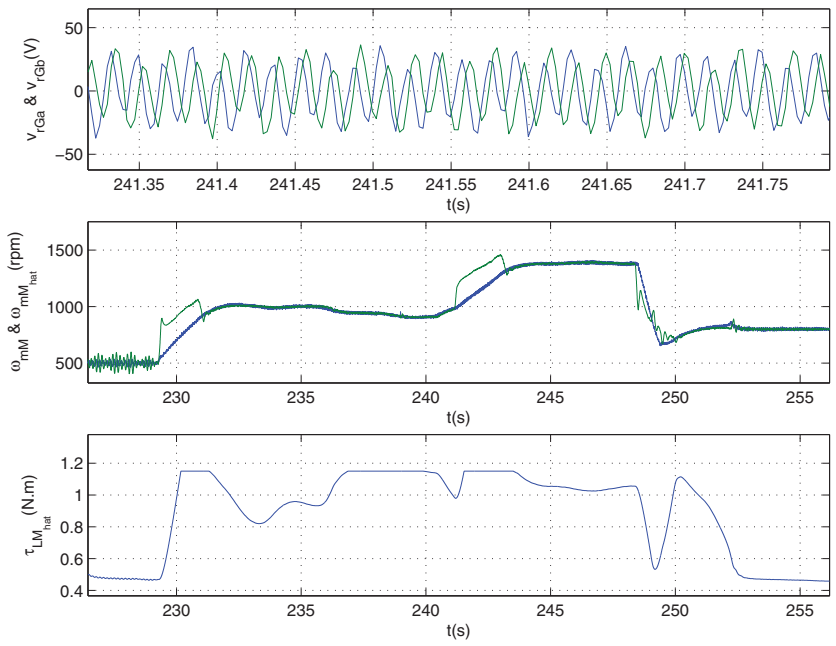

Fig. 27. PI-(a) $v_{r G a}, v_{r G b}$ (b) $\omega_{m M}, \hat{\omega}_{m M}$ (c) $\hat{\tau}_{M L}$.

These results show that the PI control alone of the stator currents is not efficient for the control of the DFIG+IM system. The PBC, with or without P or PI actions, shows much better performance.

\subsection{Robustness tests}

In order to highlight the performances of the controllers, and check their behaviour in the presence of machine parameter variations, a change in the DFIG and IM rotor and stator resistances is applied. In the real case, the resistances of a machine increase with temperature. In this case, all the resistances of the two machines used in the controllers are decreased by $40 \%$ when the "Switch on Parameters" signal value goes from 0 to 1 (see figure 31). This test has been carried out with the four controllers (i.e. PBC, PBC+P, PBC+PI and PI). The results show that all the controllers are robust to a large change in machine resistances. To be brief, only the results obtained with the PBC are reported here.

Figure 28 presents the mechanical IM speed and its smooth reference, the mechanical DFIG speed, the DFIG and IM rotor positions, the DFIG torque $\tau_{G}$ and the IM desired torque $\tau_{M d}$. The real IM speed tracks very well the reference, i.e. low overshoot and no steady state error are observed. Figure 29 shows the stator currents $i_{s a}$ and $i_{s b}$, and their references over a period of time. The stator currents do not track exactly the desired values but are bounded. This is because the goal of the PBC is to track the IM speed and to keep internal signals bounded. Figure 30 shows the DFIG rotor currents $i_{r G a}$ and $i_{r G b}$, and their references over a period of time. Again, these currents are sinusoidal and bounded.

Figure 31 presents the control signals $v_{r G a}$ and $v_{r G b}$, the rotor IM speed $\omega_{m M}$ and its estimation $\hat{\omega}_{m M}$, and the "Switch on Parameters" signal. These results illustrate the robustness of the PBC when the parameters are varied.

\section{Conclusion}

Speed-torque tracking controllers for an IM powered by a DFIG have been presented. The joint system extracts energy from a primary mechanical source that is transformed by the 

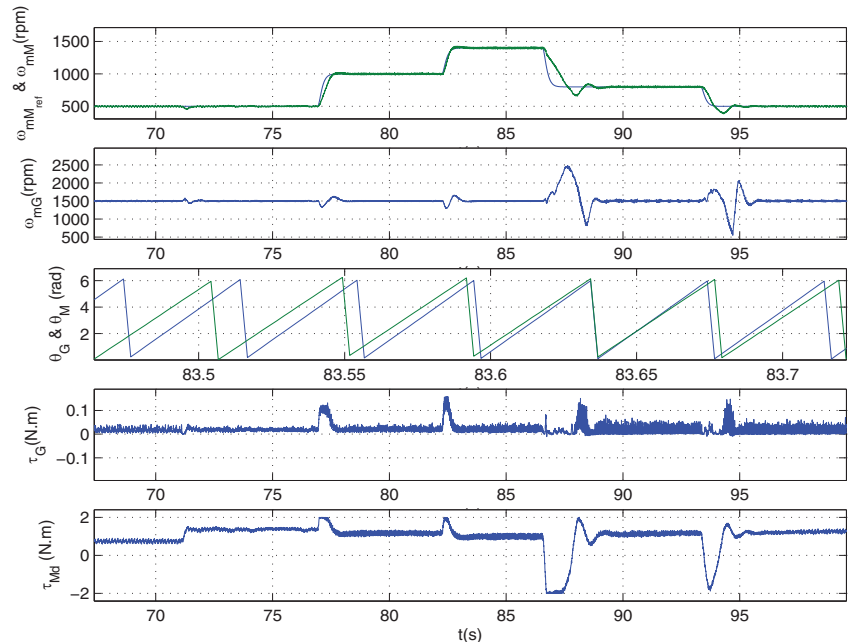

Fig. 28. PBC-robustness test-(a) Regulated Motor speed and its reference. (b)Generator speed. (c) DFIG \& IM rotor position. (d) Generator torque (e) Motor desired torque.
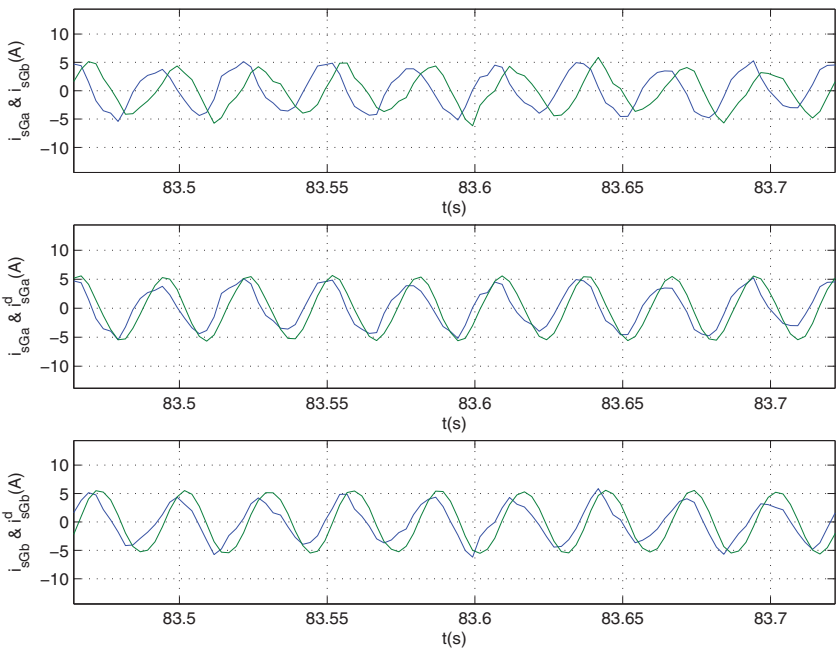

Fig. 29. PBC -robustness test-(a) $i_{s G a}, i_{s G b}$ (b) $i_{s G a^{\prime}}^{d} i_{s G a}$ (c) $i_{s G b^{\prime}}^{d} i_{s G b}$. 

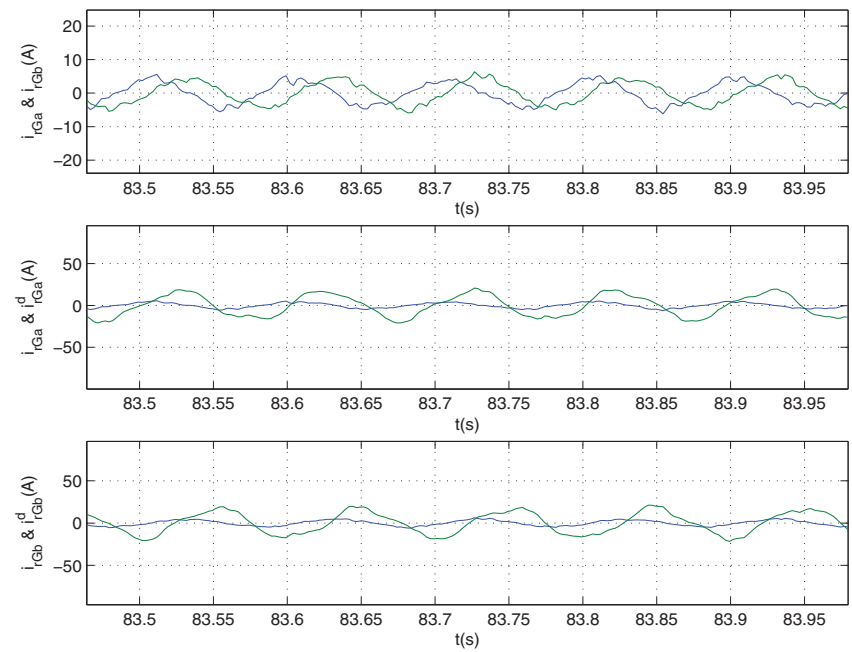

Fig. 30. PBC-robustness test-(a) $i_{r G a}, i_{r G b}$ (b) $i_{r G a^{\prime}}^{d} i_{r G a}$ (c) $i_{r G b}^{d}, i_{r G b}$.
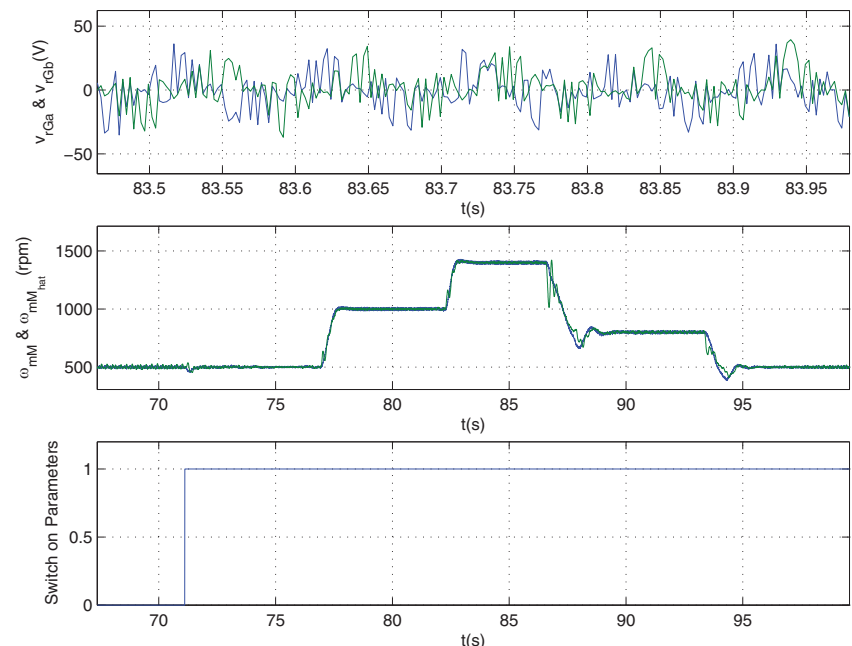

Fig. 31. PBC-robustness test-(a) $v_{r G a}, v_{r G b}$ (b) $\omega_{m M}, \hat{\omega}_{m M}$ (c) Switch.

DFIG, which at the same time controls the speed of the IM making use of the rotor voltage of the DFIG as a control variable. A complete stability proof for inner loop control is given. The proof of the overall scheme including the outer speed loop follows verbatim from (1) and is omitted here for brevity.

The main advantage of the PBC is that it requires the measurement of only two mechanical positions for the speed tracking. The PI controller applied to the inner loop provides good performance but saturation in the transient state can be observed. Robustness tests were performed to observe the behaviour of the controllers to machine parameter variations. All the proposed controllers were found to be robust towards variation in machine resistances. Also, a power flow analysis can be undertaken between the generator, the IM and the grid 
in order to optimize the efficiency of the overall system. A comparison of the experimental results of the proposed PBC, PI, PBC+P and PBC+PI algorithm is presented in Table 2. It is based on the performance obtained practically with the different controllers. In addition to the comparison criteria of Table 2, it is proposed to check the following to see what their effects are on the controllers'performance:

- $e_{\omega_{M}}=\frac{1}{n T} \sum_{i=1}^{n}\left[\omega_{M}(i)-\omega_{R e f M}(i)\right]^{2}$ indication about the IM speed tracking error. Where $n$ is the length of the sampled data and $T$ is the sampling time;

- $e_{i_{s G a}}=\frac{1}{n T} \sum_{i=1}^{n}\left[i_{s G a}(i)-i_{s G a}^{d}(i)\right]^{2}$ indication about the stator current tracking error in the phase $a$;

- Observed magnitude of $i_{s G a}$;

- $P_{\operatorname{avg}_{G}}=\frac{1}{n} \sum_{i=1}^{n}\left[\tau_{G}(i) \omega_{G}(i)\right]$ indication about the rotor average value of the instantaneous absorbed power in the DIFG;

- $P_{\operatorname{avg}_{M}}=\frac{1}{n} \sum_{i=1}^{n}\left[\tau_{M}(i) \omega_{M}(i)\right]$ indication about the rotor average value of the instantaneous absorbed power in IM;

\begin{tabular}{|l||c|c|c|c|c|c|}
\hline & $R_{S}(\Omega)$ & $R_{r}(\Omega)$ & $L_{S}(\mathrm{mH})$ & $L_{r}(\mathrm{mH})$ & $L_{m}(\mathrm{mH})$ & $J\left(\mathrm{Nm}^{2} / \mathrm{rad}\right)$ \\
\hline \hline DFIG & 0.365 & 0.559 & 0.938 & 0.938 & 12.975 & $4.358 \times 10^{-3}$ \\
\hline IM & 0.5 & 0.2 & 1.2 & 1.2 & 9.00 & $1.1 \times 10^{-3}$ \\
\hline
\end{tabular}

Table 1. The parameters for DFIG and IM

\begin{tabular}{|c|c|c|c|c|}
\hline & $\overline{P B C}$ & $\mathrm{PBC}+\mathrm{P}$ & $\mathrm{PBC}+\mathrm{PI}$ & PI \\
\hline $\begin{array}{l}\omega_{\text {RefM }} \\
{[\mathrm{rpm}]}\end{array}$ & $\begin{array}{c}500 \rightarrow 1000 \\
\rightarrow 1400 \rightarrow 800 \\
\left(1^{\text {st }} \text { order filter }\right) \\
\end{array}$ & $\begin{array}{c}500 \rightarrow 1000 \\
\rightarrow 1400 \rightarrow 800 \\
\left(1^{\text {st }} \text { order filter }\right) \\
\end{array}$ & $\begin{array}{c}500 \rightarrow 1000 \\
\rightarrow 1400 \rightarrow 800 \\
\left(1^{\text {st }} \text { order filter }\right)\end{array}$ & $\begin{array}{c}500 \rightarrow 1000 \\
\rightarrow 1400 \rightarrow 800 \\
\left(1^{\text {st }} \text { order filter }\right)\end{array}$ \\
\hline$\tau_{L M}[\mathrm{~N} . \mathrm{m}]$ & $0.5 \rightarrow 1.45 \rightarrow 0.5$ & $0.5 \rightarrow 1.4 \rightarrow 0.5$ & $0.5 \rightarrow 1 \rightarrow 0.5$ & $0.5 \rightarrow 1.15 \rightarrow 0.5$ \\
\hline $\begin{array}{l}\text { settling time } \\
\text { of } \omega_{\text {Ref } M}\end{array}$ & $0.4 s$ & $0.4 s$ & $0.4 s$ & $0.4 s$ \\
\hline $\begin{array}{l}\text { settling time } \\
\text { of } \omega_{M}\end{array}$ & $0.1 s$ & $0.1 \mathrm{~s}$ & $0.1 s$ & $2 s$ \\
\hline$\omega_{G}[\mathrm{rpm}]$ & 1500 & 1500 & 1500 & 1500 \\
\hline$e_{\omega_{M}} \times 10^{5}$ & 2.9 & 4.8 & 5.7 & 38.6 \\
\hline$e_{i_{S G Q}} \times 10^{3}$ & 3.7 & 2.65 & 2.68 & 0.25 \\
\hline $\begin{array}{c}\text { Observed magnitude } \\
\text { of } i_{S G a}[\mathrm{~A}]\end{array}$ & 5 & 7 & 8 & 10 \\
\hline$P_{\text {avg }_{G}}[\mathrm{~W}]$ & 4.7 & 5.6 & 5 & 9 \\
\hline$P_{\text {avg }_{M}}[\mathrm{~W}]$ & 58.9 & 74.4 & 70.7 & 177.9 \\
\hline
\end{tabular}

Table 2. Comparison table of experimental results

If we take in account the problem of speed tracking of the IM interconnected to the DFIG and according to the robustness tests and the experimental results presented in Table 2 we can say that the PBC controller provided the best performance.

In addition, this paper has provided the detailed analysis of operational principles of the BDFTIG. 


\section{Acknowledgment}

The authors would like to express their gratitude to Jordi Riera, Enric Fossas and Miguel Allué from IRII-UPC, Barcelona, Spain for their help with the practical experiments.

\section{References}

[1] R. Ortega, A. Loria, P.J. Nicklasson, and H. Sira-Ramirez, "Passivity-based control of Euler-Lagrange systems," in Communications and Control Engineering. Berlin,Germany:Spring-Verlag, 1998.

[2] Liu, X., G. Verghese, J. Lang and M. Önder, Generalizing the Blondel-Park Transformation of Electrical Machines: Necessary and Sufficient Conditions, IEEE Trans. Circ. Syst., Vol. 36, No. 8, pp. 1085-1067, 1989.

[3] M. Becherif, R. Ortega, E. Mendes and S. Lee, "Passivity-based control of a doubly-fed induction generator interconnected with an induction motor," in CDC 2003.

[4] M. Becherif, "Contribution aux techniques de façonnement dénergie: Application à la commande des systèmes électromécaniques," PhD thesis, Université de Paris XI, France, 2004.

[5] W. Leonhard, "Control of electrical drives," Spring-Verlag, 1985.

[6] M.S. Vicatos and J.A. Tagopoulos, "Steady state analysis of a doubly-fed induction generator under sychronous operation," IEEE Trans. on Energy Conversion, vol.4, no.3, pp.495-501, 1989.

[7] F. Bogalecka, "Dynamics of the power control of a double fed induction generator connected to the soft power grid," ISIE International Symposium on Industrial Electronics, Budapest, pp.509-513, 1993.

[8] A. Mebarki and R.T. Lipczynsky, "Novel variable speed constant frequency generation system with voltage regualtion," EPE European Conference on Power Electronics and Applications, vol.2, pp.465-471, 1995.

[9] P. Caratozzolo, E. Fossas and J. Riera "Robust nonlinear control of an isolated motion system," CIEP International Power Electronics Congress, Mexico, 2002.

[10] R. Datta and V.T. Ranganathan, "Variable-speed wind power generation using doubly fed wound rotor induction machine-a comparision with alternative schemes," IEEE Trans. on energy conversion, vol.17, no.3, pp.414-421, 2002.

[11] S. Muller, M. Deicke, and Rik W. De Doncker, "Doubly fed induction generator systems for wind turbines," IEEE Industry Applications Magazine, vol., no., pp.26-33, May/June, 2002.

[12] P. Caratozzolo, E. Fossas, and J. Riera, "Nonlinear control of an isolated motion system with DFIG," IFAC International Federation of Automatic Control , 2002.

[13] A. J. van der Schaft, " $L_{2}$-Gain and Passivity Techniques in Nonlinear Control," Springer-Verlag, Berlin, 1999. 


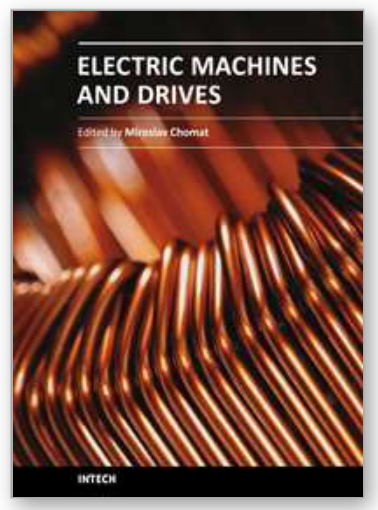

\author{
Electric Machines and Drives \\ Edited by Dr. Miroslav Chomat
}

ISBN 978-953-307-548-8

Hard cover, 262 pages

Publisher InTech

Published online 28, February, 2011

Published in print edition February, 2011

The subject of this book is an important and diverse field of electric machines and drives. The twelve chapters of the book written by renowned authors, both academics and practitioners, cover a large part of the field of electric machines and drives. Various types of electric machines, including three-phase and single-phase induction machines or doubly fed machines, are addressed. Most of the chapters focus on modern control methods of induction-machine drives, such as vector and direct torque control. Among others, the book addresses sensorless control techniques, modulation strategies, parameter identification, artificial intelligence, operation under harsh or failure conditions, and modelling of electric or magnetic quantities in electric machines. Several chapters give an insight into the problem of minimizing losses in electric machines and increasing the overall energy efficiency of electric drives.

\title{
How to reference
}

In order to correctly reference this scholarly work, feel free to copy and paste the following:

M. Becherif, A. Bensadeq, E. Mendes, A. Henni, P. Lefley and M.Y Ayad (2011). From Dynamic Modeling to Experimentation of Induction Motor Powered by Doubly-Fed Induction Generator by Passivity-Based Control, Electric Machines and Drives, Dr. Miroslav Chomat (Ed.), ISBN: 978-953-307-548-8, InTech, Available from: http://www.intechopen.com/books/electric-machines-and-drives/from-dynamic-modeling-to-experimentation-ofinduction-motor-powered-by-doubly-fed-induction-generato

\section{INTECH}

open science | open minds

\section{InTech Europe}

University Campus STeP Ri

Slavka Krautzeka 83/A

51000 Rijeka, Croatia

Phone: +385 (51) 770447

Fax: +385 (51) 686166

www.intechopen.com

\section{InTech China}

Unit 405, Office Block, Hotel Equatorial Shanghai

No.65, Yan An Road (West), Shanghai, 200040, China

中国上海市延安西路65号上海国际贵都大饭店办公楼 405 单元

Phone: +86-21-62489820

Fax: $+86-21-62489821$ 
(C) 2011 The Author(s). Licensee IntechOpen. This chapter is distributed under the terms of the Creative Commons Attribution-NonCommercialShareAlike-3.0 License, which permits use, distribution and reproduction for non-commercial purposes, provided the original is properly cited and derivative works building on this content are distributed under the same license. 